\title{
Testing the whole number interference hypothesis: contributions of inhibitory control and whole number knowledge to fraction understanding
}

\author{
Elena R. Leib ${ }^{1}$, Ariel Starr ${ }^{2}$, Jessica Wise Younger ${ }^{3}$, Project iLead Consortium, Silvia A. \\ Bunge ${ }^{1,4}$, Melina R. Uncapher ${ }^{3,5}$, Miriam Rosenberg-Lee ${ }^{6}$ \\ ${ }^{1}$ Department of Psychology, University of California, Berkeley, USA \\ ${ }^{2}$ Department of Psychology, University of Washington, Seattle, USA \\ ${ }^{3}$ Weill Institute for Neurosciences, University of California, San Francisco, USA \\ ${ }^{4}$ Helen Wills Neuroscience Institute, University of California, Berkeley, USA \\ ${ }^{5}$ Neuroscape, Department of Neurology, University of California, San Francisco, USA \\ ${ }^{6}$ Department of Psychology, Rutgers University, Newark, USA
}

Corresponding authors:

Elena R. Leib

Department of Psychology

University of California, Berkeley

eleib@berkeley.edu

Miriam Rosenberg-Lee

Department of Psychology

Rutgers University, Newark

miriam.rosenberglee@rutgers.edu

\section{CRediT author statement:}

Elena R. Leib: Conceptualization, Data curation, Formal analysis, Visualization, Writing Original Draft, Review, \& Editing. Ariel Starr: Conceptualization, Data curation, Formal analysis, Visualization, Methodology, Writing - Original Draft, Review, \& Editing. Jessica Wise Younger: Data curation, Investigation, Methodology, Project administration. Project iLead Consortium: Data curation, Funding acquisition, Investigation, Methodology, Project administration, Software. Silvia A. Bunge: Funding acquisition, Methodology, Writing - Review \& Editing. Melina R. Uncapher: Funding acquisition, Methodology, Software, Supervision. 
Miriam Rosenberg-Lee: Conceptualization, Funding acquisition, Methodology, Supervision, Writing - Original Draft, Review, \& Editing

\section{Acknowledgments:}

This research was supported by funding from the National Science Foundation, Science of Learning Collaborative Networks Grant (NSFSLCN-1540854) awarded to Melina Uncapher (lead Principal Investigator [PI]) and Adam Gazzaley, and co-PIs Joaquin Anguera, Silvia Bunge, Fumiko Hoeft, Bruce McCandliss, Jyoti Mishra, and Miriam Rosenberg-Lee. The study was performed in accordance with protocols approved by the Institutional Review Board (IRB) of the University of California San Francisco. The authors would like to thank the research staff, Jordin Rodondi, Caleb Banks, Zoe D'Esposito, John David Lorentz and the large team of UCSF volunteers as well as the students, teachers, parents, and school and district administrators who made this research program possible. The authors are also grateful to the team of developers who have made the ACE and scholastic assessments possible, including WoWLabz, Zynga.org, and Rose Feldman, and the programmers that created the aceR processing code Jose Gallegos and Monica Thieu. Correspondence should be addressed to either Elena Leib, University of California Berkeley, Berkeley, CA. Email: eleib@berkeley.edu or Miriam Rosenberg-Lee, Rutgers University, Newark, Newark, NJ. Email: miriam.rosenberglee@rutgers.edu. 


\begin{abstract}
The present study tests two predictions stemming from the hypothesis that a source of difficulty with rational numbers is interference from whole number magnitude knowledge. First, inhibitory control should be an independent predictor of fraction understanding, even after controlling for working memory. Second, if the source of interference is whole number knowledge, then it should hinder fraction understanding. These predictions were tested in a racially and socioeconomically diverse sample of US children ( $\mathrm{N}=765 ; 337$ female) in grades 3 (ages 8-9), 5 (ages 10-11), and 7 (ages 12-13) who completed a battery of computerized tests. The fraction comparison task included problems with both shared components (e.g., $3 / 5>2 / 5$ ) and distinct components (e.g., $2 / 3>5 / 9$ ), and problems that were congruent (e.g., 5/6 > 3/4) and incongruent (e.g., 3/4 > 5/7) with whole number knowledge. Inhibitory control predicted fraction comparison performance over and above working memory across component and congruency types. Whole number knowledge did not hinder performance and instead positively predicted performance for fractions with shared components. These results highlight a role for inhibitory control in rational number understanding and suggest that its contribution may be distinct from inhibiting whole number magnitude knowledge.
\end{abstract}

Keywords: fractions, inhibitory control, whole number knowledge, executive functions, rational numbers

Public significance statement: Many students struggle to master fractions, potentially because of interference from their prior whole number knowledge. Consistent with this explanation, we found that students with better inhibitory control - the ability to resolve interference - had better fraction performance. However, the results did not suggest that whole number magnitude knowledge was the source of interference. These results highlight the role of inhibitory control in developing fraction understanding in elementary and middle school. 
Rational numbers, especially fractions, are a persistent stumbling block in the elementary and middle school mathematics curriculum. Identifying the root causes of fraction difficulties is a vital step in developing instructional programs to strengthen rational number knowledge. One proposed source of challenge in understanding fractions is that many properties of whole numbers do not hold for rational numbers, and the tendency to inappropriately apply properties of whole numbers when working with rational numbers has been termed whole number bias (Ni \& Zhou, 2005). Among whole numbers, for example, larger numerals always denote larger quantities (e.g., 9>3), but this is not true for fractions (e.g., 1/9<1/3), and lower performance on fraction comparisons where whole number knowledge contradicts the appropriate rational number response is well-documented (Braithwaite \& Siegler, 2018; DeWolf \& Vosniadou, 2015; Fazio, DeWolf, \& Siegler, 2016; Gómez \& Dartnell, 2018; Meert, Gregoire, \& Noel, 2010; Obersteiner, Van Dooren, Van Hoof, \& Verschaffel, 2013; Singley \& Bunge, 2018). Implicit in this literature is what we term the whole number interference hypothesis, that whole number knowledge interferes with rational number processing, leading to the observed performance decrements. In the present study, we use an individual differences design to test two predictions that stem from this hypothesis: 1) inhibitory control supports fraction performance, and 2) whole number magnitude knowledge hinders performance.

With respect to the first prediction, if interference resolution is crucial for fraction understanding, we would expect inhibitory control - the capacity to withhold prepotent responses and resolve interference - to be a strong and independent predictor of fraction performance. Prior work has shown that executive functions, including inhibitory control and working memory - the capacity to maintain and manipulate information - support academic outcomes (Best, Miller, \& Naglieri, 2011; Lawson \& Farah, 2017; Rose, Feldman, \& Jankowski, 2011). In fact, working memory is 
the most robust predictor of mathematical outcomes in children (Bull \& Lee, 2014; Peng, Namkung, Barnes, \& Sun, 2016). Although prior rational number studies have examined the contributions of these executive functions separately (Bailey, Siegler, \& Geary, 2014; Gómez, Jiménez, Bobadilla, Reyes, \& Dartnell, 2015; Jordan et al., 2013; Matthews, Lewis, \& Hubbard, 2016; Siegler et al., 2012), there is little evidence of whether inhibitory control contributes to fraction outcomes when controlling for working memory. Establishing that inhibitory control uniquely contributes to fraction outcomes over and above working memory would bolster the claim that rational number difficulties stem, at least partly, from interference effects.

With respect to the second prediction, if the source of interference is more specifically whole number magnitude knowledge, we would expect individuals with better understanding of whole numbers to, perhaps paradoxically, perform worse on fraction tasks. This prediction therefore is a strong test of the whole number interference hypothesis, as it runs counter to the large body of research demonstrating whole number magnitude knowledge supports general math achievement (De Smedt, Noël, Gilmore, \& Ansari, 2013; Schneider et al., 2017), assessments of which often include rational number items. While whole number knowledge can refer to magnitudes, arithmetic operations, or place values, in the current study, we use the term whole number knowledge to mean knowledge about the magnitudes of whole numbers.

\section{Eliciting whole number interference in fraction comparison}

The standard approach to eliciting whole number interference in fraction comparison is to manipulate fraction pairs' congruency with whole number knowledge. This manipulation takes a different form for fraction pairs that share components (i.e., either same denominator or same numerator) versus pairs that have distinct components (i.e., neither the numerator nor denominator is the same). Among the simpler problems with shared components, same denominator pairs are 
congruent with whole number knowledge because the fraction with the larger numerator has the larger magnitude (e.g., $4 / 5>3 / 5$ ). Thus, it is possible to arrive at the correct response by using whole number comparison to select the fraction with the larger numerator. In contrast, same numerator pairs are incongruent with whole number knowledge because the fraction with the smaller denominator has the larger magnitude (e.g., 3/5>3/8). In these cases, using whole number comparison to select the fraction with the larger denominator would lead to the incorrect response. Consistent with the whole number interference hypothesis, accuracy is typically higher for congruent compared to incongruent shared component problems (Braithwaite \& Siegler, 2018; DeWolf \& Vosniadou, 2015; Fazio et al., 2016; Gómez \& Dartnell, 2018; Meert et al., 2010; Miller Singley \& Bunge, 2018).

Among the more challenging problems with distinct components, congruent pairs are typically defined as pairs in which the fraction with the largest components also has the larger magnitude (e.g., $5 / 6>3 / 4$ ); whereas in incongruent pairs, the fraction with the largest components has the smaller magnitude (e.g., 2/3 > 5/9; but see Ischebeck, Schocke, and Delazer (2009) for an exception). The assumption underlying this manipulation for distinct components is that congruent pairs should be easier than incongruent pairs because comparing either the numerators or the denominators will lead to the same correct (or incorrect) response, an effect generally born out in the literature (Gómez \& Dartnell, 2018; Gómez et al., 2015). In this study, we seek to use individual differences in inhibitory control and whole number knowledge to examine how whole number interference contributes to fraction comparison performance.

\section{Executive functions and fraction understanding}

Domain-general cognitive capacities, such as executive functions (EFs), have a well-established contribution to academic outcomes. Among the canonical EF constructs of working memory, 
inhibitory control, and cognitive flexibility (Diamond, 2013), working memory is one of the most robust predictors of mathematical outcomes (Friso-van den Bos, van der Ven, Kroesbergen, \& van Luit, 2013; Peng et al., 2016). The contributions of inhibitory control and cognitive flexibility are less clear, as many studies do not find relations with these constructs and general measures of math achievement (Lee \& Bull, 2016; Lee \& Lee, 2019; Van Dooren \& Inglis, 2015). One possible explanation for the lack of consistent effects for inhibitory control is that it may be involved primarily in mathematical domains that require resolving interference from prior knowledge.

A similar phenomenon has been found in science education, where inhibitory control predicts learning when students must undergo conceptual change but not learning of factual information (Bascandziev, Tardiff, Zaitchik, \& Carey, 2018). In fact, rational numbers have been cited as a paradigmatic example of conceptual change because learners must expand their understanding of number from discrete and countable whole numbers to continuous and dense rational numbers (Carey, 2011; Vamvakoussi \& Vosniadou, 2004). These dueling conceptions of number make rational numbers an ideal testbed for clarifying the role of inhibitory control in math outcomes.

A growing list of studies have examined correlations between inhibitory control and rational number comparison, for both fractions and decimals (Abreu-Mendoza, Coulanges, Ali, Powell, \& Rosenberg-Lee, 2020; Avgerinou \& Tolmie, 2019; Gómez et al., 2015; Ren \& Gunderson, 2021). Yet, these studies did not explicitly measure working memory. Further, although a handful of studies have looked at the role of working memory in fraction learning, it is more frequently considered a control variable rather than a variable of interest (Bailey et al., 2014; Jordan et al., 2013; Siegler et al., 2012; Starr, Leib, Younger, Uncapher, \& Bunge, 2022). One recent study involving adolescents did consider how inhibition and visual working memory relate to three factors derived from performance on a standardized math task: basic arithmetic, rational number 
transformations, and fraction arithmetic and basic algebra (Abreu-Mendoza, Chamorro, GarciaBarrera, \& Matute, 2018). Both inhibition and working memory correlated with the factors, but no regression analysis directly contrasted the contributions of these skills (Abreu-Mendoza et al., 2018). Thus, while both EF components contributed to rational number outcomes, this study did not establish whether the contribution of inhibitory control is distinct from that of working memory.

One study to date has considered contributions of all three canonical EF dimensions in a rational number comparison task. Coulanges et al. (2021) found that in college students, both working memory and inhibitory control independently predicted decimal comparison performance in counterintuitive pairs in which the decimal with the larger numerical value contained fewer digits (e.g., $0.8>0.27$ ). Interestingly, inhibitory control only predicted performance on these whole number knowledge conflicting problems, whereas working memory was also related to performance on problems that did not involve conflict (e.g., $0.80>0.27$ ), and cognitive flexibility did not contribute to either form of decimal comparison. These results highlight the centrality of working memory and inhibitory control for rational number understanding, but also point to distinctions between them. Working memory may support performance across a range of problem types, whereas inhibitory control may be especially important when intuitions based on prior knowledge, such as whole number knowledge, interfere with the correct response. In addition, the contributions of these cognitive factors may vary in younger participants who have less experience with rational numbers.

In the current study, we considered whether inhibitory control has a specific influence on incongruent fraction comparisons, which contradict prior whole number knowledge, or contributes comparably to both congruent and incongruent comparisons. If inhibitory control is needed 
exclusively for overcoming whole number magnitude knowledge, then we would expect to find an influence of inhibitory control only on incongruent pairs. On the other hand, if inhibitory control is also needed to switch between strategies on different congruency types or to inhibit whole number knowledge beyond magnitude information, then we would expect inhibitory control to contribute to both congruent and incongruent pairs. In line with this second possibility, some children may learn that fractions operate "differently" than whole numbers (Miller Singley, Crawford, \& Bunge, 2020; Rinne, Ye, \& Jordan, 2017) so they may monitor responses more carefully, therefore engaging inhibitory control on both congruency types.

Additionally, it is possible that the task used to measure inhibitory control may affect the relations to rational numbers that are found. Although the majority of studies examining inhibitory control have found positive associations with rational number outcomes (Abreu-Mendoza et al., 2020; Avgerinou \& Tolmie, 2019; Coulanges et al., 2021; Gómez et al., 2015; Ren \& Gunderson, 2021), some studies have not found significant relations between the measures (Matthews et al., 2016; Park \& Matthews, 2021; Stricker et al., 2021). Notably, Matthews and colleagues found that inhibitory control was not related to performance on a non-symbolic fraction comparison task or to conceptual understanding of rational numbers, nor was it related to symbolic fraction comparison (Matthews et al., 2016; Park \& Matthews, 2021). These conflicting results may stem from the type of inhibition task employed. While these studies (Matthews et al., 2016; Park \& Matthews, 2021) used an arrow Flanker task, studies that demonstrated relations with rational outcomes used variations of the Stroop task (Avgerinou \& Tolmie, 2019; Coulanges et al., 2021; Gómez et al., 2015) or the Hearts and Flowers task (Abreu-Mendoza et al., 2020; Ren \& Gunderson, 2021). One possible explanation for these varying results is that inhibitory control may be a diverse construct of which common inhibitory control tasks capture different subcomponents 
of this capacity. A prominent division of inhibitory constructs actually considers both Stroop and Flanker as measures of the same subcomponent, variously called "response-distractor inhibition" (distinct from resistance to proactive interference) (Friedman \& Miyake, 2004) or "interference resolution" (Younger et al., 2022). Based on this grouping, the disparate findings for Stroop and Flanker as they relate to rational number understanding are difficult to parse. However, within the rational number field a competing framework contrasts semantic inhibition, (i.e., overcoming learned knowledge) from response inhibition (i.e., overcoming prepotent responses) (Avgerinou \& Tolmie, 2019). In this view, Stroop is a measure of semantic interference, whereas Flanker can been seen as a response inhibition measure, specifically one that involves overcoming visual distractors (Lee \& Lee, 2019). Based on this division, stronger prior findings for Stroop compared to Flanker suggest that semantic interference may be the key capacity tapped by rational numbers. These conflicting organizing schemes suggest that additional work is needed to better understand how inhibitory control contributes to the development of fraction understanding.

In the current study, we selected two domain-general inhibitory control tasks, color-word Stroop task and letter Flanker. Given the diversity of measures that have been examined in relation to rational number comparison skills and the lack of clarity on which measures should have the largest contributions, we created a composite measure of these two tasks to capture individual differences in inhibitory control that are not specific to any one task. In a follow-up, supplementary analysis, we examined which measure had the stronger contribution to fraction comparison performance.

\section{Whole number knowledge: help or hindrance?}

A large body of evidence relates better symbolic whole number knowledge to higher mathematical achievement (De Smedt et al., 2013; Schneider et al., 2017). However, the whole number 
interference hypothesis suggests that in the case of fractions, better symbolic whole number knowledge may actually impede success with fraction comparison. Fractions have a bipartite structure $(a / b)$, in which the numerator and denominator enter into a multiplicative relation. This structure affords at least two different types of processing during fraction comparison: 1) holistic processing, comparing the magnitudes of the two fractions, and 2) componential processing, comparing the whole number components of the two fractions (Ischebeck et al., 2009; Meert, Gregoire, \& Noel, 2009; Miller Singley \& Bunge, 2018; Zhang, Fang, Gabriel, \& Szucs, 2014). Componential processing could create a context in which whole number knowledge bolsters performance on congruent comparisons but interferes with performance on incongruent comparisons.

Whole number knowledge is typically measured using two different types of tasks: number line estimation, in which participants estimate where a number goes on a number line, and number comparison, in which participants make a speeded judgment as to which of two numbers has the larger magnitude. Although performance on these two measures is typically correlated (Laski \& Siegler, 2007; Ramani \& Siegler, 2008), they may tap into separable aspects of whole number magnitude knowledge. Potentially, whole number line estimation may index a learner's capacity to linearly organize numerical magnitudes (Siegler, Thompson, \& Schneider, 2011). By contrast, whole number comparison may index the automaticity of accessing magnitude from numerical symbols. Because properties that apply to whole numbers do not always apply to fractions, fractions may represent a counterintuitive type of math knowledge for which this whole number automaticity, which is typically beneficial, may in fact be a hindrance (Bonato, Fabbri, Umiltà, \& Zorzi, 2007; Ischebeck et al., 2009; Rosenberg-Lee, 2021; Vamvakoussi \& Vosniadou, 2004; Zhang et al., 2014). Specifically, whole number magnitude comparison may contribute to 
componential processing during fraction comparison and thus represent a step where interference from whole numbers may come into play. For this reason, in the current study we are particularly interested in single-digit whole number comparison. We examined whether this measure predicts fraction comparison performance for congruent and incongruent problems with both shared and distinct component pairs. If whole number comparison taps the automaticity of whole number magnitude understanding, we might expect it to have a positive contribution to congruent problems but a weaker or negative contribution for incongruent problems because a focus on the magnitude of fraction components in these problems will lead to the incorrect answer. In contrast, if whole number comparison measures a deep understanding of numerical magnitude, regardless of number system (i.e., whole or rational), we would expect a positive contribution across congruency types.

Further, we examined whether these contributions differ by fraction pair component type (shared vs. distinct). On the one hand, opportunity for whole number interference is greater for distinct component problems (which require comparing multiple components). On the other hand, the presence of incongruent information may be more salient for the shared component problems. We explicitly considered whether whole number knowledge influences fraction performance over and above the contributions of working memory and inhibitory control. This approach allows us to begin separating out the need for inhibition from the automatic activation of the information to be inhibited.

\section{Tracking the development of fraction understanding}

In the United States, fractions are typically introduced in $3^{\text {rd }}$ grade, and students should have developed considerable proficiency by the end of middle school in $8^{\text {th }}$ grade (Common Core Math, 2010). Although several studies have considered the development of fraction knowledge over this time period (Kainulainen, McMullen, \& Lehtinen, 2017; Van Hoof, Degrande, Ceulemans, 
Verschaffel, \& Van Dooren, 2018), none to our knowledge have considered the changing role of cognitive building blocks like EFs or whole number knowledge, which continue to develop and improve over this age range (Constantinidis \& Luna, 2019; Opfer \& Siegler, 2007). As EFs improve, they may play a bigger role in supporting fraction performance. For example, stronger EFs may be needed more in later grades when students are implementing more sophisticated strategies. Alternatively, EFs may be more important in earlier grades when students are first learning fraction concepts. The interplay between these EFs and whole number knowledge may cause the interference effects on fraction comparison to grow as well. To investigate these relations, we analyzed cross-sectional data from $3^{\text {rd }}, 5^{\text {th }}$, and $7^{\text {th }}$ graders that were collected as part of a larger, longitudinal study (Younger et al., 2021; Younger et al., 2022). This cross-sectional sample affords a view across the first five years of fraction instruction in the US and allows us to investigate how EFs and whole number knowledge relate to fraction comparison at three different stages in the development of these skills.

\section{The current study}

The primary aim of this study was to test two a priori predictions of the whole number interference hypothesis. First, if inhibition is vital for rational number understanding, we would expect inhibitory control to be an independent predictor of fraction comparison performance after accounting for working memory. Second, if difficulties with rational numbers stem from whole number magnitude interference, then we would expect whole number comparison to be negatively associated with fraction comparison performance. For both predictions, we explored how fraction pair congruency type (congruent or incongruent with whole number knowledge) influenced the relations between the cognitive factors. We also examined whether inhibitory control and whole number knowledge interacted to determine whether participants with poorer whole number 
knowledge require less inhibition. Finally, we explored whether the relations between inhibitory control, whole number knowledge, and fraction comparison were stable across grades or changed over development. Together, these analyses paint a comprehensive picture of the role of inhibitory control and whole number knowledge during the crucial early years of the development of fraction understanding.

\section{Methods}

\section{Project iLead Study}

A total of $1,2803^{\text {rd }}$ through $8^{\text {th }}$ grade children participated in the Project iLead study, which was a longitudinal study over two school years (2016-2017 and 2017-2018) that investigated EF development in elementary and middle school (Younger et al., 2021). Participants were recruited from nine schools in northern California - seven public schools in one district (5 elementary K-5; 2 middle 6-8), one parochial K-8 school, and one private K-8 school. Of the seven public schools, two of the elementary schools and one of the middle schools were Title 1 schools, a designation under US law indicating that these schools had high percentages of students from low-income families and received federal funds to support these students.

Participants were assessed in the fall and spring of each school year, for a total of four assessment periods. Each assessment period consisted of two sessions, one for the EF assessments and a second for an academic performance assessment. This current study focuses only on year 1 of the study, when students from grades 3, 5, and 7 were recruited (see Younger et al. (2021) for more details about study enrollment).

\section{Participants}

The data described here are from 765 students who provided sufficient data for our tasks of interest in the first year of the project. The sample was ethnically and socioeconomically diverse (Table 
1). The study was performed in accordance with the Internal Review Board at [REDACTED]. Written parental or guardian consent was obtained from all participants at the beginning of the study, and verbal assent from all participants was obtained before all in-class data collection sessions. At the end of the study, all students in participating classrooms received snacks and stickers, regardless of their individual participation and performance.

\section{Table 1}

Demographic characteristics of the sample. See Supplementary Methods for details on the sample size difference between grades. See Table S1 for age at testing separated by timepoint.

\begin{tabular}{lccc} 
& Grade 3 & Grade 5 & Grade 7 \\
n=180 & $\mathbf{n}=\mathbf{1 6 3}$ & $12.63(11.30,14.59)$ \\
\hline Age at testing (years) & $8.80(7.99,10.34)$ & $10.61(9.83,11.72)$ & $0.5 \%$ \\
$\quad$ Not reported & $4.4 \%$ & $3.7 \%$ & \\
Gender & & & $46.0 \%$ \\
F & $47.8 \%$ & $35.0 \%$ & $47.4 \%$ \\
M & $38.9 \%$ & $44.8 \%$ & $6.6 \%$ \\
Not reported & $13.3 \%$ & $20.2 \%$ & \\
Ethnicity & & & $1.2 \%$ \\
American Indian or Alaskan Native & $0.0 \%$ & $0.0 \%$ & $31.0 \%$ \\
Asian & $38.3 \%$ & $26.4 \%$ & $1.2 \%$ \\
Black or African American & $2.8 \%$ & $3.7 \%$ & $0.2 \%$ \\
Blank on Purpose & $0.0 \%$ & $0.0 \%$ & $7.8 \%$ \\
Filipino & $3.3 \%$ & $6.1 \%$ & $31.0 \%$ \\
Hispanic or Latino & $18.3 \%$ & $23.9 \%$ & $0.7 \%$ \\
Pacific Islander & $1.7 \%$ & $0.0 \%$ & $4.3 \%$ \\
Two or More Races & $5.6 \%$ & $4.3 \%$ & $15.9 \%$ \\
White & $16.7 \%$ & $15.3 \%$ & $6.6 \%$ \\
Not reported & $13.3 \%$ & $20.2 \%$ & $33.9 \%$ \\
Free/Reduced Lunch & $27.2 \%$ & $33.7 \%$ & $6.6 \%$ \\
Not reported & $13.3 \%$ & $20.2 \%$ & \\
\hline Mean (Range); & & & \\
\hline
\end{tabular}

Mean (Range); \% 


\section{Procedure}

Two groups of assessments were administered to participants at two timepoints during each school year, once in the fall and again in the spring. The first group of assessments was the Adaptive Cognitive Evaluation (ACE) battery (Younger et al., 2021), which consisted of nine EF tasks and was administered at each timepoint. The second group were scholastic assessments and were divided into two subsets (seven to eight tasks each), one of which included the numerical comparison tasks. Each subset was administered to participants once per year in alternating semesters. Classrooms were randomly assigned to complete each task set in either the fall or spring of each year, with the other subset of assessments administered at the other timepoint. At each of the timepoints, ACE was administered first, and the scholastic assessments were administered approximately six weeks later $(M=5.7$ weeks, $\min .=1.9$, max. $=10)$. All tasks were administered on iPads in a group setting $(M=30$ students, $\min .=7$, $\max .=83)$ at schools during approximately 50 minute sessions. Tasks were designed to be as short as possible given the number of assessments that needed to fit into each of the sessions. The number of facilitating researchers ranged from four to 12 , depending on group size, and administration took place in various school contexts, including classrooms, libraries, cafeterias, and gymnasiums. Instructions were given verbally to the whole group by the lead facilitator, with complementary visual instructions on a 24 " x 36 " color flipbook in front of the class. All participants in a group began each task at the same time. When every participant had completed the task, the lead facilitator provided instructions for the next task. Before completing each task, participants completed a few practice trials and could ask the researchers questions. Researchers monitored the session throughout. 


\section{Executive function tasks}

\section{Working memory tasks}

The forward spatial span task was adapted from the Corsi Block Task (Corsi, 1972) for use on touch-screen tablets. Participants had to reproduce a spatial sequence of illuminated locations. The backward spatial span task had the same design as forward spatial span, but participants were prompted to recall the sequence in the reverse order. For both tasks, participants started at a span of 3, which is a common starting point for Corsi Block Tasks for children in grades 3 and above (Farrell Pagulayan, Busch, Medina, Bartok, \& Krikorian, 2006). The longest attempted span was used as the outcome measure. For more details on these tasks, see Supplementary Methods and Figure S1.

\section{Inhibitory control tasks}

The color-word Stroop task was a modified computerized version of the Stroop paradigm (Stroop, 1935) for manual responses (Mead et al., 2002). It consisted of 50 experimental trials, $70 \%$ congruent (font color matches color word) and 30\% incongruent (font color differs from color word). The letter Flanker task, which used the letters "A" through "D", was a computerized adaption of the original Eriksen and Eriksen (1974) task that also used these letters. The task consisted of 50 experimental trials, of which $50 \%$ were incongruent and $50 \%$ were congruent. Response windows for both tasks were adaptive to keep the tasks challenging for all ages of participants (see Younger et al. (2021) for more details about adaptivity). In part due to this adaptivity, rate correct score (RCS) of the full task, a measure of speed-accuracy tradeoff, was used as the outcome measure for these tasks. RCS is calculated by dividing the total number of correct responses by the total response time in seconds across correct and incorrect trials (Vandierendonck, 2017; Woltz \& Was, 2006), and can be thought of as the number of correct 
responses per unit time. For more details on these tasks, including why we selected RCS as the outcome measure, see Supplementary Methods and Figure S2.

\section{Numerical comparison tasks}

For the whole number and fraction comparison tasks, participants always saw two numbers, one on the left side of the screen and one on the right, and were instructed to tap the larger of the two numbers. Within each task and level, the position of the larger number (i.e., on the left or right) was counterbalanced and trials were presented in a random order. Trials that were not answered within the response window were marked as incorrect. Whole number comparison was administered first, followed by fraction comparison.

\section{Whole number comparison}

In this task, the stimuli were two single-digit whole numbers, and all pairs had a magnitude difference of 1 (see Figure S3). Thus, the task had eight different comparison problems - 1 versus

2, 2 versus 3, and so on, up to 8 versus 9. Each problem was presented four times, twice with the larger number on the left and twice with the larger number on the right, yielding 32 trials. However, due to a programming error, participants only saw 30 trials. At the fall timepoint, participants were missing the two presentations of 7 versus 8 with the larger digit on the right. At the spring timepoint, participants were missing two trials with the larger digit on the right, one presentation of 6 versus 7 and one of 7 versus 8 . The response window was three seconds. 


\section{Fraction comparison}

We designed this task to have two levels of difficulty (Figure 1) because this study included a broad range of grades and therefore a broad range of expected fraction knowledge. Level 1 consisted of 16 unique shared component trials: eight congruent, same denominator problems (e.g., 4/9 vs 7/9) and eight incongruent, same numerator problems (e.g., 3/4 vs 3/7). In half of the trials for each congruency type, the larger number was presented on the left. In selecting fraction pairs for the congruency types, we controlled for the average magnitude distance between the two fractions and the partial distance (either 1 or 3 ) between the digits in the non-shared component. Further, fractions were limited to those that could not be reduced to lower terms (see Table S2 for a full list of stimuli and additional properties).

To prevent students from feeling discouraged by seeing problems beyond their ability level (e.g., material they were not yet expected to know based on the national math standards), only participants who achieved at least $75 \%$ accuracy (i.e., $\geq 12$ problems correct) moved on to Level

Level 1 Shared Components

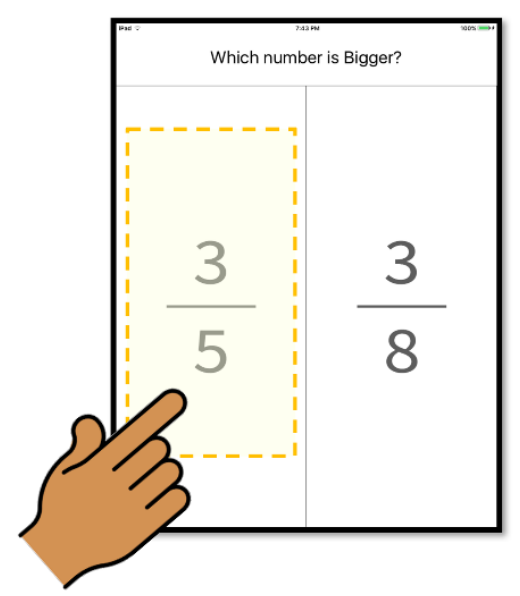

Level 2 Distinct Components

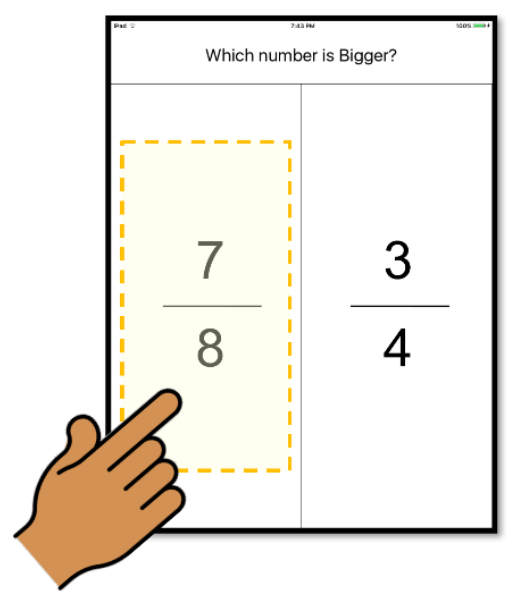

Figure 1. Two sample trials from the fraction comparison task. In Level 1 (left), the fraction pair had shared components, either the same denominator or the same numerator. In Level 2 (right), the fraction pair had distinct components. If participants reached $75 \%$ accuracy on Level 1 , they advanced to Level 2 . The response window for both levels was 4 seconds. The hands and yellow boxes are for illustrative purposes only and indicate the correct response option for each trial. 
2. Participants who did not meet this mark completed Level 1 again, though we only analyzed data from their first time through the task. $75 \%$ accuracy on Level 1 was chosen as the criterion in order to ensure that participants who advanced had some mastery of Level 1 (i.e., reliably more accurate than chance at 50\%) without excluding too many participants (i.e., including only those at ceiling) in order to ensure sufficient variability on Level 2.

Level 2 consisted of 10 unique problems with distinct components (e.g., 3/4 vs 5/6). Each problem was presented twice to counterbalance which fraction in the pair was presented on the left, yielding 20 trials. Half of the trials were congruent with whole number knowledge, such that the fraction with the larger numerator and denominator had the larger magnitude (e.g., 3/7 vs 4/8). The other half were incongruent with whole number knowledge, such that the fraction with the larger numerator and denominator had the smaller magnitude (e.g., 2/3 vs 5/9). In selecting fraction pairs for the congruency types, we again controlled for the magnitude distance between the two fractions. We were also able to control for the partial distance for the numerators (which ranged from 1-3 for both congruent and incongruent), but mathematically (Rosenberg-Lee, 2021) this means we could not control for denominator distance (which ranged from 1-3 for congruent and 3-6 for incongruent pairs) and other features (see Table S3 for a full list of stimuli and their properties).

For both levels, we chose a response window of four seconds to encourage automatic processing rather than strategies that involve calculation, such as computing the cross products. Examination of the fraction comparison data suggest that the 4 second response window was sufficient because the average time until a correct response was made was 1.88 seconds for Level 1 and 1.99 seconds for Level 2. Further, only $15 \%$ of incorrect trials on either level were due to no responses (i.e., timing out). 


\section{Data analysis}

\section{Preparation for analysis}

First, trial- and task-level data were cleaned based on the procedures outlined in the Supplementary Methods. After following these procedures, we created composite measures for working memory (WM) from performance on the forward and backward span tasks and for inhibitory control (IC) from performance on the Stroop and Flanker tasks. To compute the composite measures, participants' scores on the EF tasks were first z-scored within grade and timepoint to account for the possibility of scores increasing over the school year. If participants had data for both tasks for each construct (WM: $99.35 \%$ of participants; IC: $95.56 \%$ of participants), their composite score consisted of the average of the two standardized scores. If participants had data for only one task, their composite score corresponded to the standardized score for that task (WM: $0.52 \%$ forward span only and 0.13\% backward span only; IC: 1.57\% Flanker only and 2.88\% Stroop only). Participants with no data for either task were excluded from analysis. The composite measures were then z-scored within each grade and timepoint. For the follow-up analyses of the independent contributions of Stroop and Flanker, only participants with data for both tasks were included in the analysis (see Supplementary Methods).

Only participants who scored above chance $(50 \%)$ on whole number comparison were included in analyses (24 participants excluded), as we reasoned that participants who scored below chance likely either did not understand the instructions or chose not to follow them. Whole number comparison accuracy was z-scored within grade and timepoint, to account for differences in the stimuli and so that all predictors were standardized, for subsequent modelling.

Next, because we used fraction comparison congruency type (congruent or incongruent) as a fixed effect in the mixed effects models, we needed to ensure that participants had enough trials of each 
type to analyze. Therefore, we included in our analyses only participants who had data for at least $75 \%$ of the trials for each congruency type after trial-level data cleaning (Fraction Comparison Level 1: $\geq 6$ trials of each type, excluded 13 participants; Fraction Comparison Level 2: $\geq 8$ trials of each type, excluded 18 participants).

Finally, participants who had data for all of our primary tasks of interest (Fraction Comparison Level 1, WM, IC, and Whole Number Comparison) were included in the final sample of 765 participants (Table 2). Given that at least 75\% accuracy on Fractions Level 1 was required to advance to Level 2, a subset of this final sample had data for Level $2^{1}$. The sample with Level 2 data consisted of 473 participants (Table 2).

\section{Table 2}

Sample sizes by grade level and fraction comparison component type. In order to complete fraction comparison with distinct components (Level 2), participants needed to achieve $75 \%$ accuracy on fraction comparison with shared components (Level 1). The percentage of participants within each grade that advanced to Level 2 is shown in parentheses.

\begin{tabular}{lcccc}
\hline Fraction Comparison & Grade 3 & Grade 5 & Grade 7 & Total \\
\hline $\begin{array}{l}\text { Shared components } \\
\text { (Level 1) }\end{array}$ & 180 & 163 & 422 & 765 \\
$\begin{array}{l}\text { Distinct components } \\
\text { (Level 2) }\end{array}$ & $55(31 \%)$ & $98(60 \%)$ & $320(76 \%)$ & $473(62 \%)$ \\
\hline
\end{tabular}

\section{Analysis methods}

We applied mixed effect models to examine the relationship between the predictor variables and grade with fraction comparison performance. In all models, we implemented grade as an ordered

\footnotetext{
${ }^{1}$ Due to the need for participants to have enough trials of each congruency type to be included in the analyses for each level, a small number of participants $(\mathrm{N}=6)$ scored above $75 \%$ on Level 1 and completed Level 2 but were excluded from the Level 1 analyses for insufficient trials of each congruency type. These participants were still included in the Level 2 analyses because they did have the sufficient number of trials for each congruency type at that level. The results did not change if the 6 participants were excluded from the Level 2 analyses. See the Supplementary Methods for additional details about how many trials for each congruency type were considered sufficient.
} 
factor (Grade 3, Grade 5, Grade 7), which allowed us to maintain the rank order of increasing grade levels while also acknowledging that grade cannot be treated as a continuous variable. Therefore, the regression models used a polynomial contrast, which outputs linear and quadratic effects for grade and interactions with grade. Implementing grade as an ordered factor allowed us to capture differences in performance across grades that may be better characterized by a quadratic compared to a linear function, for example in the case of floor effects in lower grades or ceiling effects in higher graders.

For our main analyses, we employed logistic mixed effects models predicting fraction comparison trial-level accuracy. All mixed effects models had the same fixed and random effects structure: fixed effects of grade level (Grade 3, Grade 5, Grade 7), congruency type (congruent or incongruent), and their interaction, plus a random intercept for participant and a random slope for congruency type. We will refer to this model as the base model.

To this base model, we first added the WM composite score and then added the IC composite score. Model comparison between the WM model and the WM + IC model addressed the question of whether IC is an independent predictor of fraction comparison accuracy, explaining additional variance over and above WM. We then added whole number comparison and compared this model to the WM + IC model to understand the relation between whole number comparison and fraction comparison after accounting for WM and IC. Successive models were compared with likelihood ratio tests using the anova function (lmerTest). To explore possible interactions between the predictors of interest, we also constructed a model of fraction comparison accuracy with interactions between grade, congruency type, IC, and whole number comparison. In cases where this model indicated significant interactions, we computed follow-up analyses to better understand 
the sources of interactions. Finally, we ran follow-up analyses to examine Stroop and Flanker as independent predictors of fraction accuracy (see Supplementary Methods).

Because we used logistic mixed effects regressions predicting accuracy, the resulting linear models specified the log odds of getting a trial correct. However, it is more interpretable to describe the results in terms of the odds by exponentiating the beta coefficients $\left(e^{\beta_{x}}\right)$, rather than keeping it in terms of the $\log$ odds. For slope coefficients, $e^{\beta_{x}}$ gives the odds ratio, relative to 1 . When $e^{\beta_{x}}>$ $1,\left(e^{\beta_{x}}-1\right) \times 100$ gives the percent increase in the odds of getting a trial correct for every 1 -unit increase in the predictor. When $0<e^{\beta_{x}}<1,\left(1-e^{\beta_{x}}\right) \times 100$ represents the percent decrease in the odds for every 1-unit increase in the predictor.

\section{Transparency and openness}

This study's design and its analysis were not pre-registered. The data analyzed in this study represent a subset of the data collected, and data from the other measures will be reported elsewhere [REDACTED]. We report all data exclusions and all manipulations for the tasks of interest. The tasks and data are available from [REDACTED] by request. The data will be made publicly available two years after publication. Data cleaning and analysis scripts are available at [REDACTED]. All analyses were conducted in R 3.6.2 (R Core Team, 2019). We used the lmerTest package to conduct the mixed effects models (Kuznetsova, Brockhoff, \& Christensen, 2017) and ggplot, version 3.3.3 (Wickham, 2016) and source code from the raincloudplots package (Allen et al., 2021) to visualize the data. 


\section{Results}

\section{Effect of grade on predictor and outcome measures}

\section{Executive functions and whole number comparison}

Performance on each of the EF predictor measures improved with grade (Figure 2A-D; see Supplementary Results for details of the linear regression analyses). Preliminary analyses of accuracy and response time on Stroop and Flanker showed the expected congruency effects (Table S4; Figure S4), and RCS was used as the outcome measure for these tasks for the rest of the analyses. For whole number comparison, accuracy ranged from an average of $92 \%$ in $3^{\text {rd }}$ grade to $95 \%$ in $7^{\text {th }}$ grade. Despite this limited range, a linear model predicting accuracy on this task revealed a significant linear effect of grade $(B=0.02, S E=0.003, p<.001)$, which indicates that accuracy on whole number comparison increased linearly with grade, with no quadratic effect $(B=-$ $0.005, S E=0.004, p=.242$ ), see Figure 2E. 
A

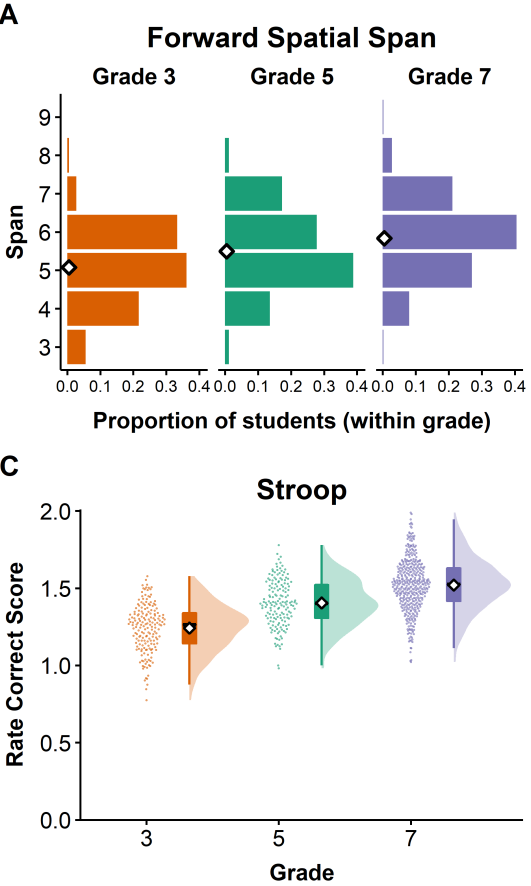

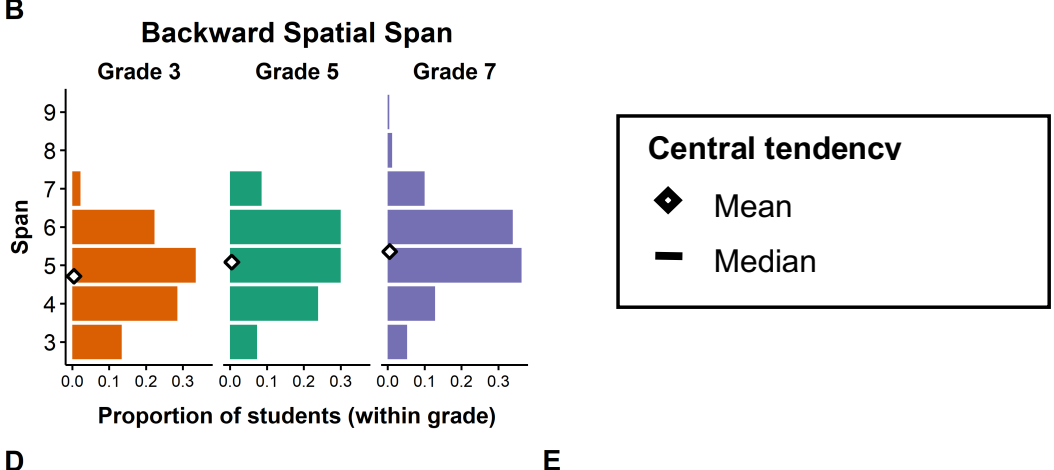

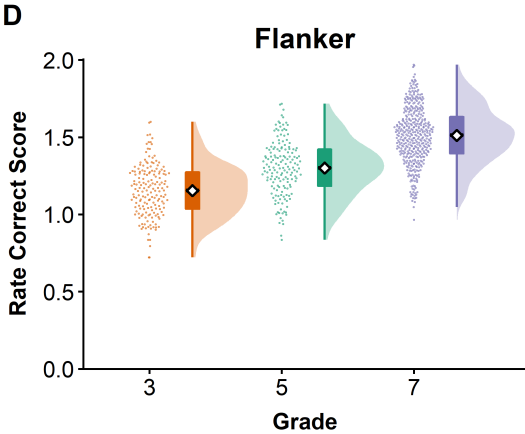

Figure 2. Distribution of performance by grade for the two working memory tasks, (A) forward and (B) backward spatial span, the two inhibitory control tasks (C) color-word Stroop and (D) letter Flanker, and (E) whole number comparison. Diamonds indicate mean and black horizontal lines indicate medians. If the mean and median values are very close together, the diamond occludes the horizontal line.

\section{Fraction comparison with shared components (Level 1)}

For fraction comparison with shared components (Level 1), congruent problems have the same denominator and incongruent problems have the same numerator. On average, participants in all three grades performed above chance $(50 \%)$ on both congruency types $\left(t \mathrm{~s}>3.90, p_{\mathbf{s}}<.001\right.$; see Figure 3). To test the effects of grade on accuracy, we employed the logistic mixed effects base model described previously: fixed effects of grade level (Grade 3, Grade 5, Grade 7), congruency type (congruent or incongruent), and their interaction, with a random intercept for participant and a random slope for congruency type. This model revealed a conditional effect of grade $(B=1.11$, $S E=0.11, p<.001)$, which reflects the expected increase in accuracy with grade, and a conditional effect of congruency type ( $B=-0.74, S E=0.08, p<.001)$, which reflects the expected lower accuracy for same numerator problems compared to same denominator problems. There was also a significant quadratic effect of grade $(B=-0.35, S E=0.13, p=.007)$, which significantly interacted 
Fraction Comparison with Shared Components

A

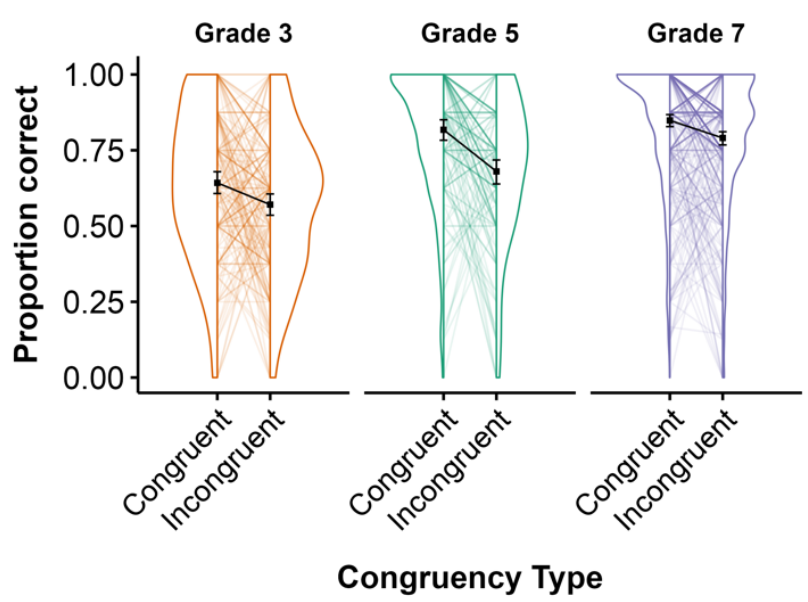

B

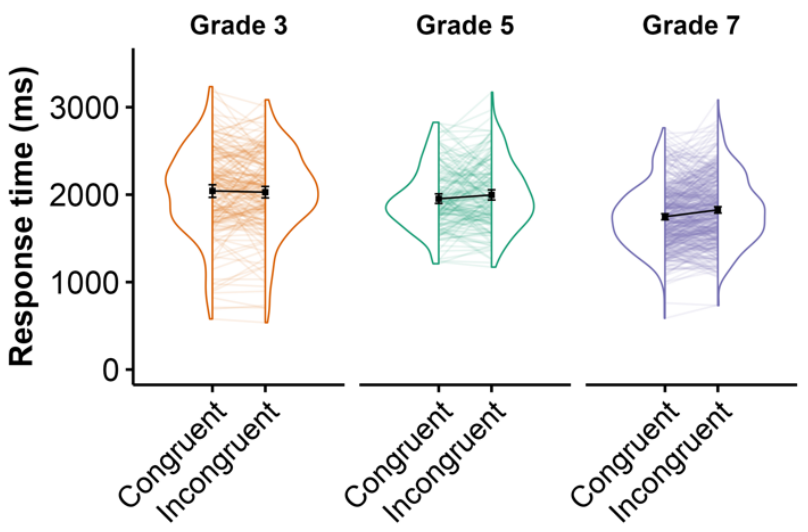

Congruency Type

Figure 3. (A) Accuracy and (B) response time distributions for fraction comparison with shared components (Level 1). Congruent problems have shared denominators and incongruent problems have shared numerators. Each colored line connects a participant's averages on congruent and incongruent problems. The black points represent the overall mean, and the black lines connect these means. Error bars indicate the bootstrapped $95 \%$ confidence intervals around the means.

with congruency type $(B=0.41, S E=0.14, p=.003)$, reflecting plateauing accuracy for same denominator problems in grade 5 versus a linear increase in accuracy for same numerator problems across grades 3, 5, and 7 (see Figure $\mathrm{S} 5$ for an illustration of the grade effects).

To examine the effect of grade on RTs, we employed a linear mixed effects model with the same fixed and random effects structure predicting RTs on correct trials. This model revealed a conditional effect of grade $(B=-220.18, S E=24.88, p<.001)$, which reflects the the expected linear decrease in RTs with increasing grade. Overall, participants were slower on same numerator problems than on same denominator problems $(B=75.23, S E=14.22, p<.001$, Figure 3$)$, and there was a significant interaction between the linear grade effect and congruency type ( $B=68.41$, $S E=23.17, p=.003$, see Figure S5). Post-hoc grade-wise Tukey comparisons showed that this effect was driven by slower RTs for same numerator relative to same denominator trials in grades 5 (Estimated Marginal Mean $(E M M)=-97.9, S E=27.3, p=.005)$ and $7(E M M=-112.3, S E=16.2$, $p<.001)$, but no difference in grade $3(E M M=-15.5, S E=28.5, p=.994)$. 


\section{Fraction comparison with distinct components (Level 2)}

Contrary to our expectation, participants performed better on incongruent compared to congruent fraction comparison problems with distinct components (Level 2). A logistic mixed effects model predicting accuracy from the previously described base model showed a conditional effect of congruency type $(B=1.18, S E=0.11, p<.001$; see Figure 4$)$, confirming that performance on incongruent problems was more accurate than on congruent problems, and there were no conditional effects of grade $(B \mathrm{~s}=0.11$ and 0.09 for linear and quadratic effects, respectively, $p \mathrm{~s}>$ 0.366). Further, there was a significant interaction between congruency type and linear effect of grade $(B=0.54, S E=0.18, p=.004)$. Given that congruent was the reference category, the lack of conditional effect of grade but presence of an interaction indicates no improvement across grade for congruent problems and a linear improvement across grade for incongruent problems (see Figure S6 for clearer illustration of grade effects).

A linear mixed effects model predicting RTs on correct Level 2 trials revealed that participants were also faster on incongruent problems than on congruent problems $(B=-111.21, S E=26.02$,

Fraction Comparison with Shared Components

A

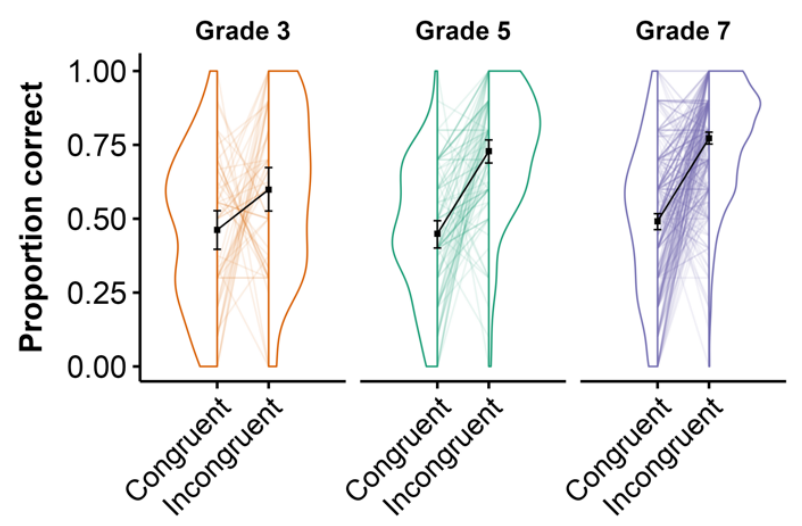

Congruency Type
B

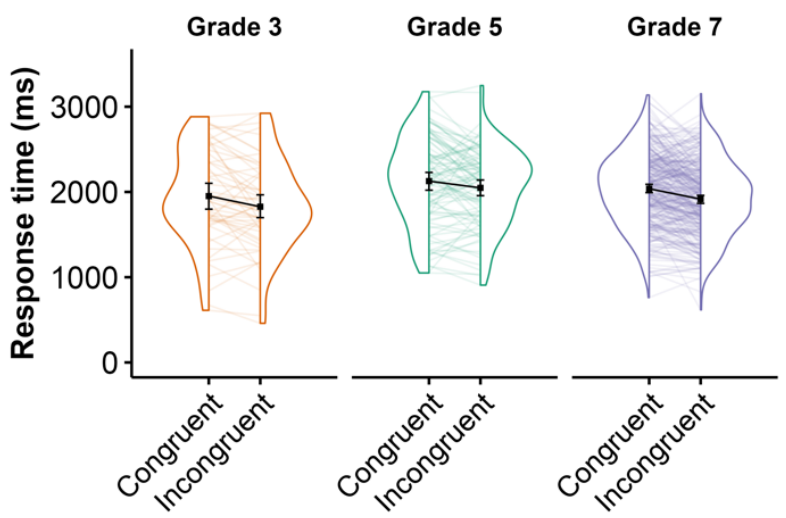

Congruency Type

Figure 4. (A) Accuracy and (B) response time distributions for fraction comparison with distinct components (Level 2). Each colored line connects a participant's averages on congruent and incongruent problems. The black points represent the overall mean, and the black lines connect these means. Error bars indicate the bootstrapped $95 \%$ confidence intervals around the means. 
$p<.001$, Figure 4). There was also a significant quadratic effect of grade $(B=-136.16, S E=53.27$, $p=.011$ ), capturing the inverted-U shaped pattern of increasing RTs from grades 3 to 5 and decreasing RTs from grades 5 to 7 (Figure S6).

\section{Predicting performance on fraction comparison with shared components (Level 1)}

In the next series of analyses, we investigated how the three predictors of interest - WM, IC, and whole number comparison - related to accuracy on fraction comparison with shared components (Level 1). Given the significant correlations between most of our variables of interest (Figure 5A), we employed a series of logistic mixed effects models to directly address our primary research questions: namely, the independent contributions of IC and whole number knowledge on fraction comparison ability. Building on the base model, we successively added the WM composite score, the IC composite score, and standardized whole number comparison accuracy.

The model including WM explained the data better than the base model $\left(\chi^{2}(1)=44.83, p<.001\right)$. Every increase in WM score of one standard deviation was associated with a $34 \%$ increase in the odds of getting a trial correct (Table 3). The model adding IC better explained the data than the model with only WM as a covariate $\left(\chi^{2}(1)=54.60, p<.001\right)$, indicating that IC was an independent predictor of fraction comparison accuracy, over and above WM. Every increase in IC score of one standard deviation was associated with a $41 \%$ increase in the odds of getting a trial correct (Table 3, Figure 5B). With the inclusion of IC, WM remained a significant predictor, indicating that the two types of EF measures make independent contributions to fraction performance. The model that additionally included whole number comparison explained the data better than the model with only WM and IC as covariates $\left(\chi^{2}(1)=40.45, p<.001\right)$, showing that whole number knowledge predicts fraction accuracy after taking WM and IC into account. Every increase in whole number comparison accuracy of one standard deviation was associated with a $31 \%$ increase in the odds of 
getting a trial correct (Table 3, Figure 5C). This series of analyses suggested that working memory, inhibitory control, and whole number comparison are all unique predictors of fraction comparison accuracy.

\section{Table 3}

Beta coefficients for logistic mixed effects models predicting accuracy on fraction comparison with shared components (Level 1) from our predictors of interest. The base model includes fixed effects of grade level $(3,5,7)$, congruency type (congruent or incongruent), and their interaction, plus a random intercept for participant and a random slope for congruency type. WM=Working Memory Composite Score, IC=Inhibitory Control Composite Score, and WNC $=$ Whole Number Comparison. Congruent problems had the same denominator and incongruent problems had the same numerator. (See Table S5 for 95\% confidence intervals)

\begin{tabular}{lllll}
\hline & Base & Base + WM & $\begin{array}{l}\text { Base }+ \text { WM + } \\
\text { IC }\end{array}$ & $\begin{array}{l}\text { Base + WM + } \\
\text { IC + WNC }\end{array}$ \\
\hline Grade (Linear) & $1.11^{* * *}$ & $1.10^{* * *}$ & $1.10^{* * *}$ & $1.12^{* * *}$ \\
Grade (Quadratic) & $-0.35^{* *}$ & $-0.35^{* *}$ & $-0.35^{* *}$ & $-0.36^{* *}$ \\
Congruency Type & $-0.74^{* * *}$ & $-0.74^{* * *}$ & $-0.74^{* * *}$ & $-0.75^{* * *}$ \\
Grade (Linear) * Congruency Type & -0.21 & -0.20 & -0.20 & -0.21 \\
Grade (Quadratic) * Congruency Type & $0.41^{* *}$ & $0.41^{* *}$ & $0.41^{* *}$ & $0.41^{* *}$ \\
Working Memory & & $0.29^{* * *}$ & $0.16^{* * *}$ & $0.13^{* *}$ \\
Inhibitory Control & & & $0.34^{* * *}$ & $0.30^{* * *}$ \\
Whole Number Comparison & & & & $0.27 * * *$ \\
AIC & & 11735.67 & 11683.07 & 11644.62 \\
BIC & 11778.50 & 11809.45 & 11764.23 & 11733.16 \\
\hline
\end{tabular}

$* * * \mathrm{p}<0.001 ; * * \mathrm{p}<0.01 ;{ }^{*} \mathrm{p}<0.05$

To determine if there were any interactions between grade, congruency type, IC, and whole number comparison, we constructed a model with the four-way interaction and the corresponding three- and two-way interactions (see Table S6). WM was included as a single covariate with no interaction terms. This model revealed a significant three-way interaction between the linear effect of grade, congruency type, and whole number comparison $(B=-0.34, S E=0.12, p=.006)$, as well as 
three two-way interactions (congruency type * whole number comparison: $B=0.18, S E=0.08$, $p=.018$; linear effect of grade * whole number comparison: $B=0.29, S E=0.11, p=.009$; and quadratic effect of grade * congruency type: $B=0.36, S E=0.14, p=.011$ ). Notably, none of these interactions included IC.

To examine these interactions with whole number comparison more closely, we implemented separate logistic mixed effects models for each grade. These grade-wise models included congruency type, whole number comparison, and their interaction, as well as WM and IC as covariates, and the same random effects structure described previously (Table 4). Performance on same numerator problems was significantly worse than on same denominator trials for all grades. For $3^{\text {rd }}$ graders, there was a significant interaction between congruency type and whole number comparison but no conditional effect of whole number comparison; specifically, whole number comparison did not predict accuracy on same denominator problems, but positively predicted accuracy on same numerator problems (Figure 5A and C). For $5^{\text {th }}$ graders, although Figure 5A shows a positive correlation between whole number comparison and accuracy for both congruency types, it seems that WM and IC accounted for that relation, as there was no conditional effect of whole number comparison nor an interaction with congruency type. Finally, for $7^{\text {th }}$ graders, better whole number comparison predicted better accuracy on both congruency types (Table 4). These follow up analyses suggest that whole number knowledge may play a different role in fraction comparison across different grades and levels of fraction experience.

Because IC was a robust predictor of fraction performance on Level 1, we conducted a series of follow-up analyses to more closely examine the two measures of IC: Stroop and Flanker (see Supplementary Methods, Figure S7A, and Table S8). Both Stroop and Flanker individually explained variance in fraction comparison after accounting for $\mathrm{WM}\left(\chi^{2}=26.72, p<.001\right.$ and 
$\chi^{2}=37.46, p<.001$, respectively). Further, Stroop and Flanker both explained variance in fraction comparison after additionally accounting for the other inhibitory control measure $\left(\chi^{2}=10.96\right.$, $p<.001$ and $\chi^{2}=21.69, p<.001$, respectively). These analyses suggest that both measures are independent predictors of fraction comparison accuracy for shared component problems. 


\section{Predictors of Fraction Comparison Performance Shared Components (Level 1)}

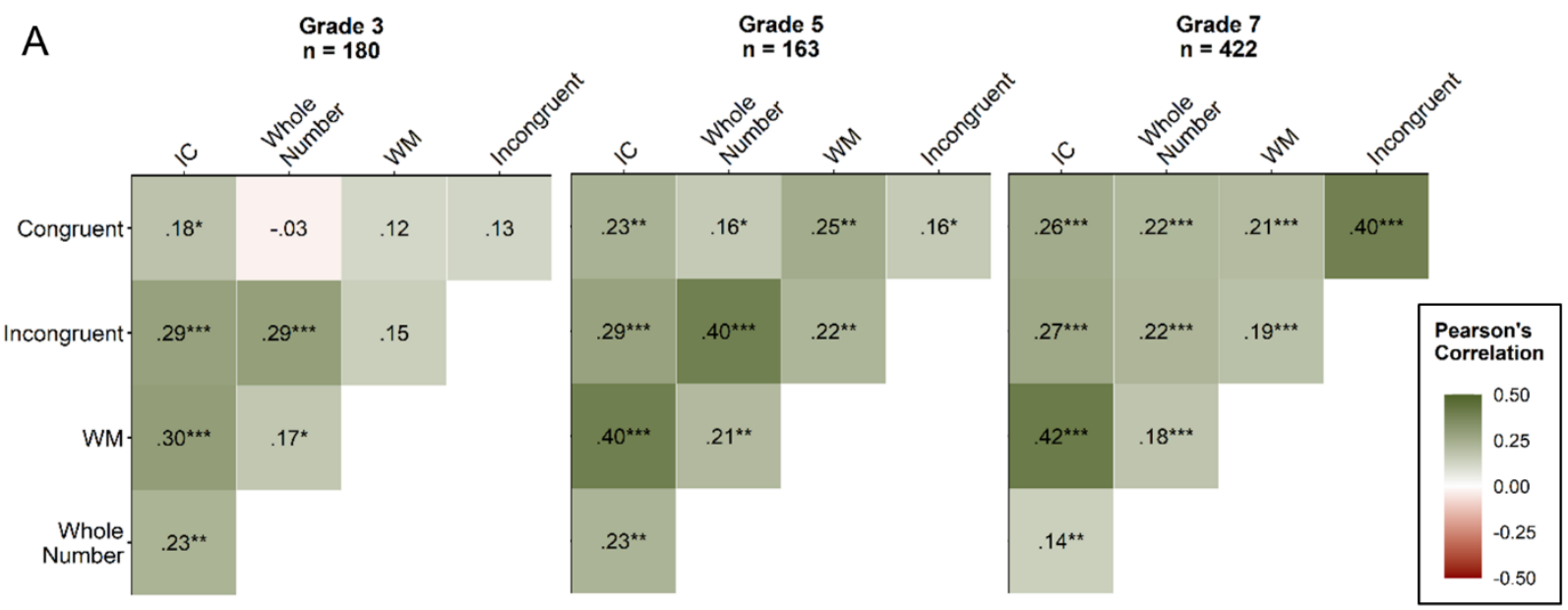

B
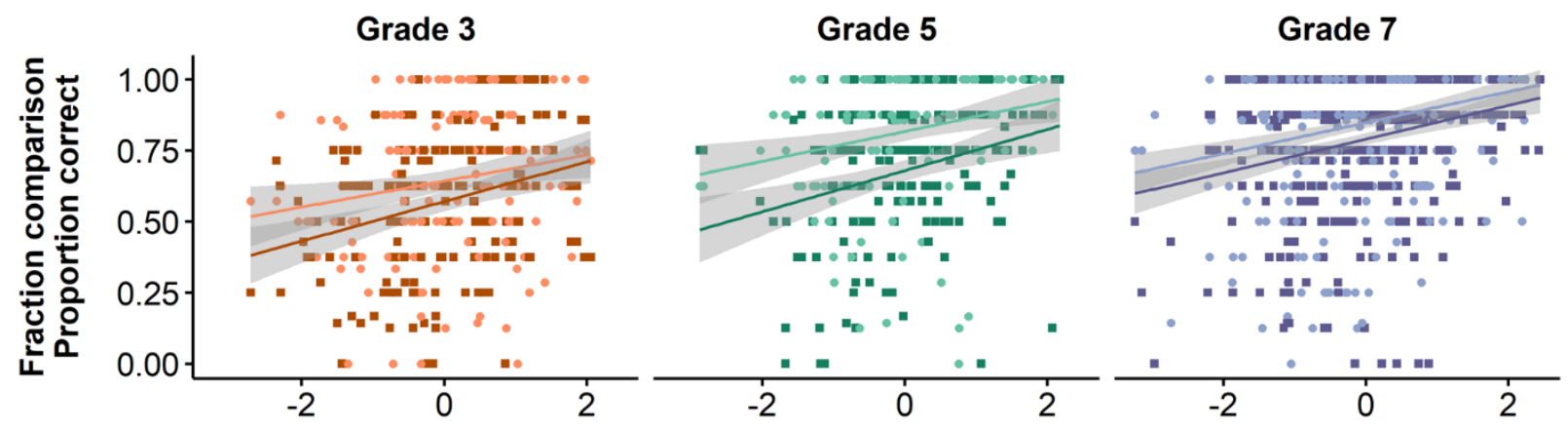

Inhibitory Control Composite Score
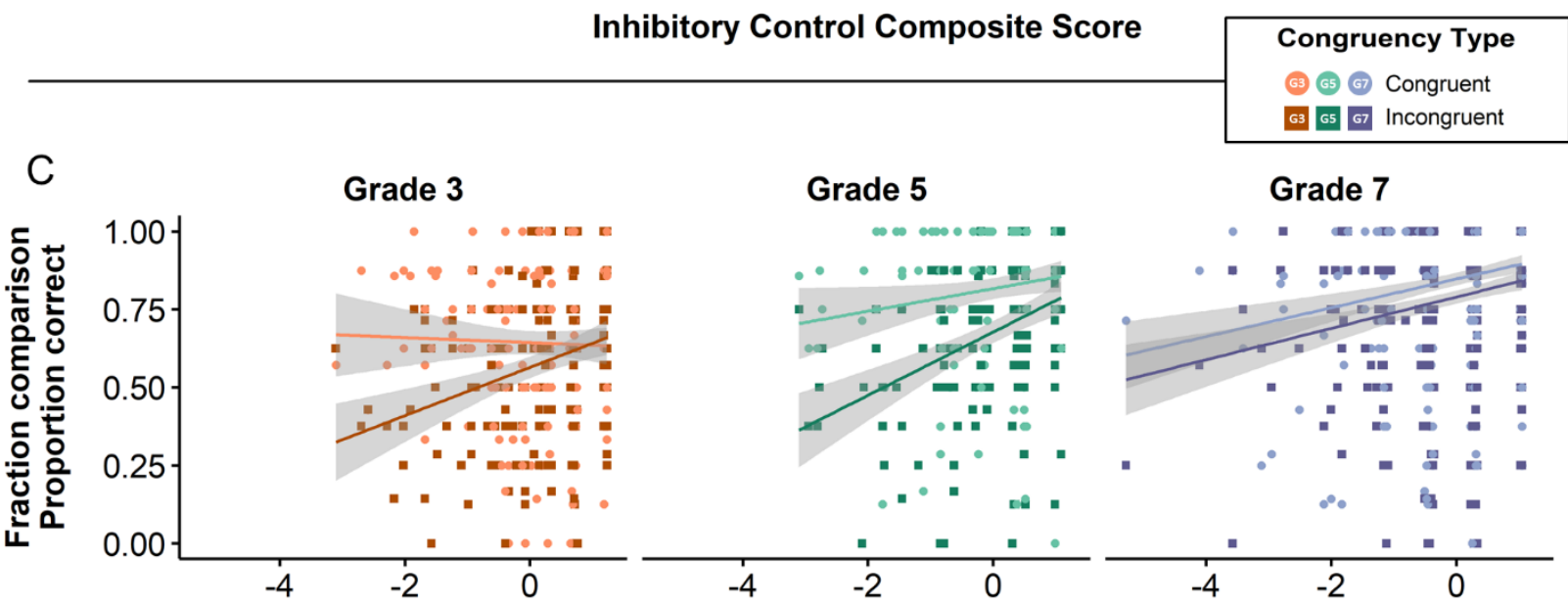

Whole Number Comparison (normed proportion correct)

Figure 5. (A) Zero-order Pearson correlation coefficients between fraction comparison with shared components (Level 1) accuracy on each congruency type and the predictors of interest: working memory composite score (WM), inhibitory control composite score (IC), and whole number comparison accuracy. Uncorrected p-values: ${ }^{* * *} \mathrm{p}<0.001 ;{ }^{* *} \mathrm{p}<0.01 ;{ }^{*} \mathrm{p}<0.05$ (B) Relation between inhibitory control composite score and accuracy for congruent and incongruent trials. (C) Relation between whole number comparison and accuracy for congruent and incongruent trials. For (B) and (C), points and lines are shaded by congruency type, with the lighter color points and lines representing congruent (same denominator) problems and the darker color points and lines representing incongruent (same numerator) problems. 


\section{Table 4}

Beta coefficients for grade-wise logistic mixed effects models predicting accuracy on fraction comparison with shared components (Level 1) from our predictors of interest. (See Table S7 for 95\% confidence intervals)

\begin{tabular}{llll}
\hline & Grade 3 & Grade 5 & Grade 7 \\
\hline Congruency Type & $-0.42^{* * *}$ & $-1.07^{* * *}$ & $-0.78^{* * *}$ \\
Working Memory & 0.07 & $0.18^{*}$ & 0.13 \\
Inhibitory Control & $0.24^{* * *}$ & $0.26^{* *}$ & $0.35^{* * *}$ \\
Whole Number Comparison & -0.11 & 0.21 & $0.36^{* * *}$ \\
Congruency Type & $0.39^{* *}$ & 0.21 & -0.10 \\
Whole Number Comparison & 3555.24 & 2577.36 & 5498.35 \\
AIC & 3608.60 & 2629.86 & 5559.41 \\
BIC & & & \\
\hline
\end{tabular}

$* * * \mathrm{p}<0.001 ; * * \mathrm{p}<0.01 ;{ }^{*} \mathrm{p}<0.05$

\section{Predicting performance on fraction comparison with distinct components (Level 2)}

Next, we examined the predictors of performance on fraction comparison with distinct components (Level 2) with a parallel set of analyses to those carried out for Level 1. Only participants who achieved at least $75 \%$ accuracy on Level 1 moved onto Level 2 . By this criterion, $31 \%$ of $3^{\text {rd }}$ graders $(55 / 180), 60 \%$ of $5^{\text {th }}$ graders $(98 / 163)$, and $76 \%$ of $7^{\text {th }}$ graders $(320 / 422)$ advanced to Level 2 (Table 2). A chi-squared test for trend in proportions indicated that the proportion of participants advancing to Level 2 increased with grade $\left(\chi^{2}(1)=107.34, p<.001\right)$.

After considering the correlations between our predictor variables and performance on pairs with distinct components (Figure 6A), we more formally tested for independent contributions of IC and whole number comparison to fraction comparison. Again, we started with the base model and then conducted a series of logistic mix effects models adding additional predictors (Table 5). The model including WM did not explain the data better than the base model $\left(\chi^{2}(1)=2.61, p=.106\right)$, indicating 
that WM was not a significant predictor of fraction accuracy with distinct components. Next, IC was added to the model, and this model explained the data better than the model with only WM as a covariate $\left(\chi^{2}(1)=12.83, p<.001\right)$, indicating that participants with higher IC scores also performed better on this level. Every increase in IC of one standard deviation was associated with a $15 \%$ increase in the odds of getting a trial correct (Table 5, Figure 6B). However, adding whole number comparison to the model did not explain the data better than the model with only WM and IC as covariates $\left(\chi^{2}(1)=0.08, p=.772\right)$, as whole number comparison was not a significant predictor of fraction accuracy at this level (Table 5, Figure 6C). This series of analyses suggests that for the more difficult fraction comparison problems with distinct components (Level 2), only individual differences in IC predicted accuracy.

To determine whether there were any interactions between grade, congruency type, IC, and whole number comparison, we again constructed a model with the four-way interaction and the corresponding three- and two-way interactions (see Table S10). WM was included as a single covariate with no interaction terms. This model confirmed a significant interaction between linear effect of grade and congruency type $(B=0.61, S E=0.23, p=.007)$, which was already captured in the base model. While visual inspection of Figure $6 \mathrm{~B}$ suggests that IC had a larger influence on fraction comparison in grades 5 and 7 than 3, the two-way interaction between quadratic effect of grade and IC did not reach significance $(B=0.13, S E=0.14, p=.361)$. Thus, the results of this interaction model did not warrant grade-wise follow-up analyses. 


\section{Predictors of Fraction Comparison Performance Distinct Components (Level 2)}

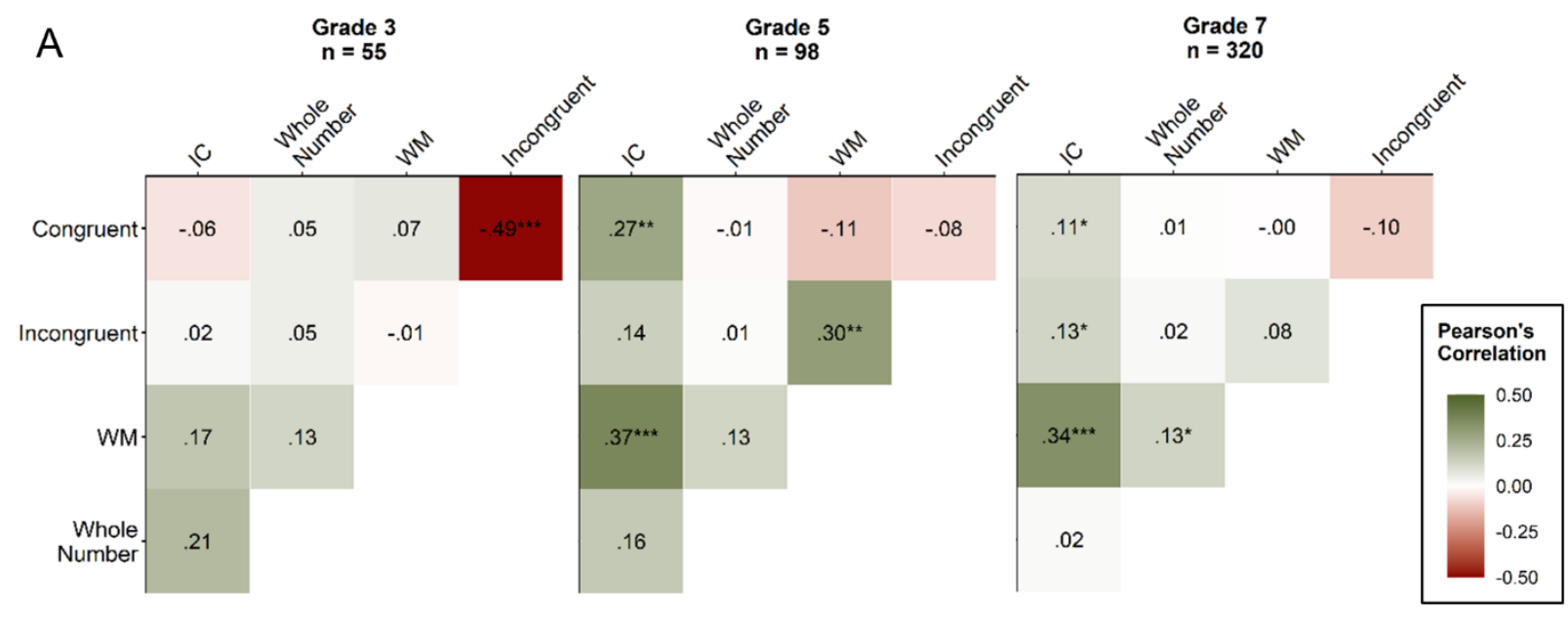

B
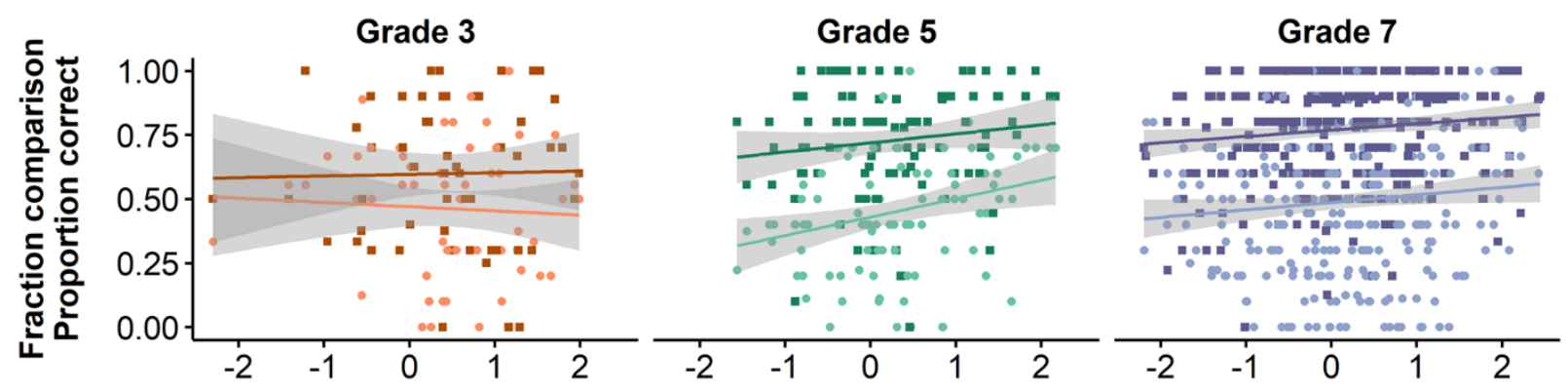

Inhibitory Control Composite Score
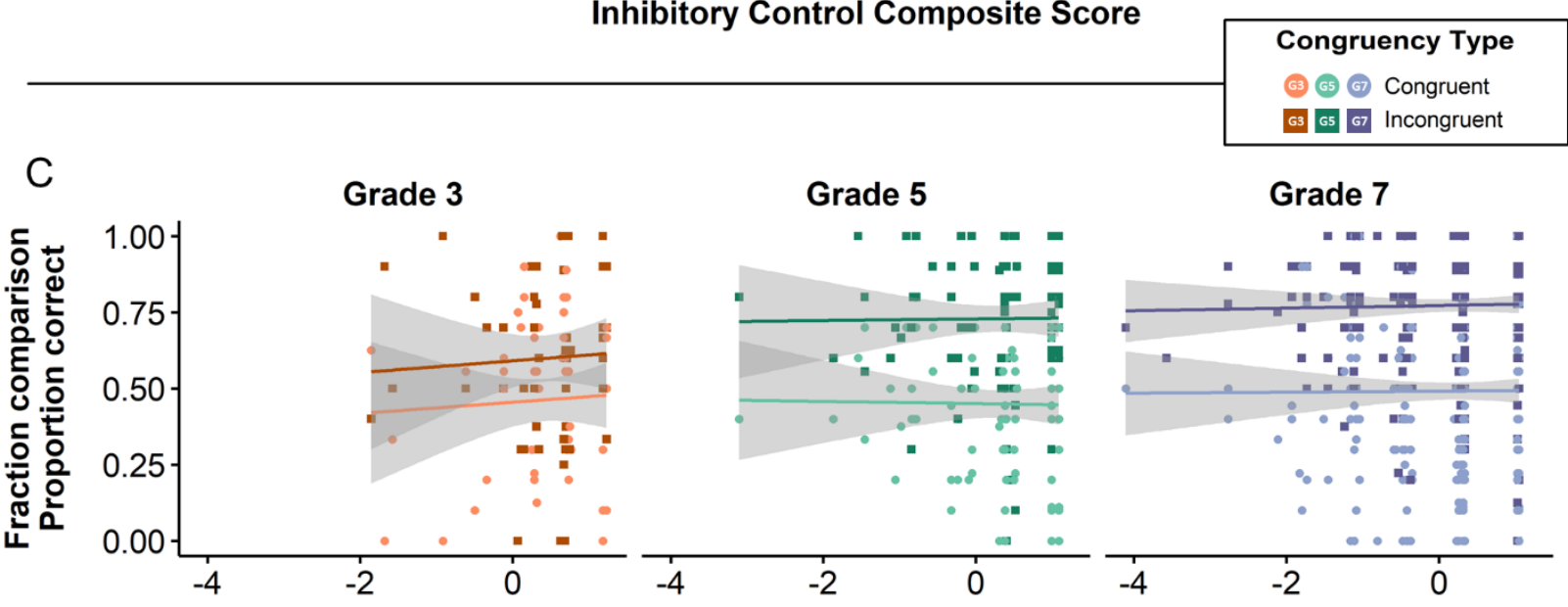

Whole Number Comparison (normed proportion correct)

Figure 6. (A) Zero-order Pearson correlation coefficients between fraction comparison with distinct components (Level 2) accuracy on each congruency type and the predictors of interest: working memory composite score (WM), inhibitory control composite score (IC), and whole number comparison accuracy. Uncorrected p-values: ${ }^{* * *} \mathrm{p}<0.001 ; * * \mathrm{p}<0.01 ; * \mathrm{p}<0.05$ (B) Relation between inhibitory control composite score and accuracy for congruent and incongruent trials. (C) Relation between whole number comparison and accuracy for congruent and incongruent trials. For (B) and (C), points and lines are shaded by congruency type, with the lighter color points and lines representing congruent problems and the darker color points and lines representing incongruent problems. 
Because IC was a robust predictor of fraction performance on Level 2, we conducted a series of follow-up analyses to more closely examine the two component measures of IC: Stroop and Flanker (see Supplementary Methods, Figure S7B, and Table S11). These analyses revealed that both Stroop and Flanker explained variance in fraction comparison after accounting for WM $\left(\chi^{2}=10.27, p=.001\right.$ and $\chi^{2}=7.40, p=.007$, respectively). Further, Stroop explained variance in fraction comparison with distinct components over and above Flanker $\left(\chi^{2}=6.02, p=.014\right)$. However, Flanker did not explain variance in fraction performance after accounting for Stroop $\left(\chi^{2}=3.14, p=.076\right)$. Thus, including Stroop and Flanker as separate predictors revealed an independent contribution to explaining distinct component fraction comparison accuracy for Stroop, but not for Flanker.

\section{Table 5}

Beta coefficients for logistic mixed effects models predicting accuracy on fraction comparison with distinct components (Level 2) from our predictors of interest. The base model includes fixed effects of grade level $(3,5,7)$, congruency type (congruent or incongruent), and their interaction, plus a random intercept for participant and a random slope for congruency type. WM=Working Memory Composite Score, IC=Inhibitory Control Composite Score, and WNC $=$ Whole Number Comparison. (See Table S9 for 95\% confidence intervals)

\begin{tabular}{lllll}
\hline & Base & Base + WM & $\begin{array}{l}\text { Base }+ \text { WM }+ \\
\text { IC }\end{array}$ & $\begin{array}{l}\text { Base + WM + } \\
\text { IC + WNC }\end{array}$ \\
\hline Grade (Linear) & 0.11 & 0.11 & 0.14 & 0.14 \\
Grade (Quadratic) & 0.09 & 0.10 & 0.09 & 0.09 \\
Congruency Type & $1.18^{* * *}$ & $1.18^{* * *}$ & $1.18^{* * *}$ & $1.18^{* * *}$ \\
Grade (Linear) * Congruency Type & $0.54^{* *}$ & $0.53^{* *}$ & $0.54^{* * *}$ & $0.54^{* *}$ \\
Grade (Quadratic) * Congruency Type & -0.25 & -0.25 & -0.25 & -0.25 \\
Working Memory & & 0.06 & 0.01 & 0.01 \\
Inhibitory Control & & & $0.14^{* * *}$ & $0.14^{* * *}$ \\
Whole Number Comparison & & & & 0.01 \\
AIC & 11033.33 & 11032.72 & 11021.89 & 11023.81 \\
BIC & 11097.45 & 11103.96 & 11100.26 & 11109.30 \\
\hline
\end{tabular}


$* * * \mathrm{p}<0.001 ; * * \mathrm{p}<0.01 ; * \mathrm{p}<0.05$

\section{Discussion}

In this study, we tested two predictions of the whole number interference hypothesis as it relates to fraction comparison in a large, diverse sample of elementary and middle school students. Consistent with the prediction that if part of students' difficulty with mastering fractions comes from the need to inhibit knowledge about whole numbers that does not apply to fractions, we found that individual differences in inhibitory control related to fraction comparison performance. However, we did not find support for the prediction that students with stronger whole number knowledge would experience more interference, and thus, counterintuitively, perform worse on fraction comparison than students with weaker whole number knowledge. These results held for fraction comparisons with both shared and distinct components. Together, our findings point to a pivotal role for inhibitory control in rational number understanding that may be distinct from inhibiting whole number magnitude knowledge.

\section{Development of fraction comparison performance for $3^{\text {rd }}$ to $7^{\text {th }}$ grade}

The youngest participants in our study were in $3^{\text {rd }}$ grade, the grade in which fractions are commonly introduced into the mathematics curriculum in the United States (Common Core Math, 2010). As would be expected based on increasing mastery of fractions with age and accumulating instructional experience, we found that participants became more accurate and responded more quickly on fraction comparison with shared components (Level 1) across grades. As expected, performance on congruent (same denominator) problems exceeded performance on incongruent (same numerator) problems. Further, performance on congruent problems leveled off after $5^{\text {th }}$ grade, whereas performance on incongruent problems increased linearly across grades. However, for the more difficult, distinct component problems (Level 2), contrary to expectation, performance 
was worse for the congruent than incongruent problems. Further, performance did not improve for congruent problems but did increase linearly across the grades for incongruent problems. Therefore, though there was general improvement across grades, consideration of congruency type revealed subtle performance differences that will be discussed in the following sections.

\section{Inhibitory control supports fraction comparison across grades and problem difficulty}

The first aim of this study was to assess the role of inhibitory control in supporting fraction understanding. Although previous studies have documented the contribution of working memory (Jordan et al., 2013) or inhibitory control (Avgerinou \& Tolmie, 2019; Gómez et al., 2015) to fraction comparison performance, none, to our knowledge, have explored the simultaneous contribution of these abilities. We found that when fractions had shared components (Level 1), both working memory and inhibitory control each explained unique variance, and that when fractions had distinct components (Level 2), only inhibitory control explained unique variance. Statistically, this relationship did not interact with grade, although visual inspection suggests it was stronger in grades 5 and 7 than grade 3 . A larger sample of $3^{\text {rd }}$ graders may be needed to detect the development of reliance on inhibitory control for distinct component fraction comparison. Nevertheless, these results are in line with a general prediction of the whole number interference hypothesis, namely that inhibitory control should be a vital capacity for rational number understanding.

The strong view of the whole number interference hypothesis, however, would predict that inhibitory control would be especially important for solving fraction comparisons incongruent with whole number knowledge. This prediction was not born out in the present study, as there was an equal contribution of inhibitory control to performance on both congruency types. Prior studies of rational number comparison have found significant contributions of inhibitory control for 
incongruent but not congruent problems in proportional reasoning (Abreu-Mendoza et al., 2020; Coulanges et al., 2021) and decimal comparison (Coulanges et al., 2021). However, these studies did not explicitly test for the difference in contribution between congruency types, making it unclear whether the contribution of inhibitory control is greater for incongruent than congruent problems. Negative priming studies of fraction comparison also suggest a specific role of inhibition for incongruent comparisons (Fu, Li, Xu, \& Zeng, 2020; Rossi, Vidal, Letang, Houdé, \& Borst, 2019). These studies show that solving an incongruent, same numerator problem decreases performance on a subsequent congruent, same denominator problem relative to a control prime trial. Notably, these studies involved only shared component problems, making it unclear whether this priming effect extends to distinct component problems where participants might not even know to inhibit their whole number knowledge. Further, they do not consider the effects of congruent priming on incongruent performance, which might be expected if inhibitory control is needed for both problem types. Interestingly, one recent study looked at this question using a block switching design (Van Hoof, Ceulemans, \& Van Dooren, 2021) and found that switching from congruent to incongruent fractions incurred the same switch cost as the reverse switch, relative to solving the same problem type twice (stay trials). This pattern of results is consistent with our finding that inhibitory control is needed when working with fractions, regardless of congruency type. An interesting area for future work would be to add an inhibitory control assessment to a negative priming study to examine the relation between differences in switch costs and individual variation in this crucial capacity. Another area for future work would be to examine whether the pattern of relations between inhibitory control, congruency type, and performance holds across different types of rational numbers or whether there are fundamental differences between types. 
In contrast to the consistent contributions of inhibitory control for both shared and distinct component problems, the role of working memory differed by component type. Working memory had a significant contribution to shared component (Level 1) performance but was not a significant predictor of distinct component performance (Level 2), regardless of whether inhibitory control was present in the model. One explanation is that working memory may support the transition to more automatic processing for easier fraction comparisons (as with shared components), whereas for more challenging tasks (as with distinct components), the ability to inhibit prior knowledge and resolve interference is what matters most. An alternative possibility is that this null effect may stem from the fact that only the strongest performing participants (who included participants with higher working memory) and mostly the oldest participants $\left(5^{\text {th }}\right.$ and $7^{\text {th }}$ grade students who have been learning about fractions for the longest and also have the highest working memory) moved on to Level 2, resulting in a smaller sample size and a possible selection bias. Future studies should use a more fine-grained measure of working memory, such as the half step span measure employed in Coulanges et al., (2021), or a more sensitive task to test working memory's involvement in fraction comparison. Nevertheless, the finding that inhibitory control is a significant predictor of distinct component fraction comparison performance when controlling for working memory further highlights the importance of inhibitory control for fraction understanding. We also found that the contribution of inhibitory control was stable across grades, counter to the possibility that it only becomes important when participants understand that they should be inhibiting their whole number knowledge. This result suggests that even at the earliest stages of fraction understanding, inhibitory control plays a role.

A final question related to inhibitory control afforded by this data set was assessing the contribution of different experimental measures. Prior work has found associations between 
various Stroop tasks (i.e., numerical and color-word) and rational number outcomes (Avgerinou \& Tolmie, 2019; Coulanges et al., 2021; Gómez et al., 2015), and also the Hearts and Flowers task (Abreu-Mendoza et al., 2020; Ren \& Gunderson, 2021). In fact, the arrow Flanker task is the only measure of inhibitory control studied so far that has not been found to be associated with rational number performance (Matthews et al., 2016; Park \& Matthews, 2021). In the current study, both color-word Stroop and letter Flanker were collected. Based on the prior studies and the classification of Stroop as a semantic inhibition task (Avgerinou \& Tolmie, 2019), we might have expected that Stroop would have stronger predictive power than Flanker. Consistent with this prediction, for distinct component pairs (Level 2) only Stroop was significant when both were included in the regression analyses, despite both measures predicting performance when entered alone. For shared component pairs (Level 1), both measures predicted performance when entered alone. However, when entered together, the Flanker task had the stronger predictive power, although Stroop remained significant.

Although both color-word (Coulanges et al., 2021) and numerical Stroop (Gómez et al., 2015) tasks have been previously employed in the literature, here we used the color-word version, which is a domain-independent measure of EF (e.g., it does not involve processing numerical magnitudes), to avoid circularity in interpretations. However, numerical Stroop tasks, which explicitly involve inhibiting whole number magnitude knowledge, may index the automaticity with which numerical magnitude information is accessed (Bugden \& Ansari, 2011), making it a potentially useful diagnostic tool to identify students at risk for fraction difficulties. In sum, these results suggest that a wide range of inhibitory control measures can predict rational number outcomes, and more research is warranted to establish if a specific task is more predictive of certain aspects of rational number knowledge and performance, especially in the classroom context. 


\section{Whole number knowledge supports some forms of fraction comparison}

The second aim of this study was to examine the counterintuitive prediction that better knowledge of whole number magnitudes could be detrimental to fraction understanding. Because properties of whole numbers do not always apply to fractions - for example, the presence of a larger numeral does not always imply the larger fraction magnitude — students who are more able to automatically access whole number magnitudes may experience greater interference when resolving fraction magnitudes, resulting in poor fraction comparison performance. However, this prediction runs contrary to the large body of evidence showing that whole number knowledge is positively related to a variety of math learning outcomes, both when measured concurrently and as a predictor of future learning (De Smedt et al., 2013; Schneider et al., 2017).

Consistent with the view that whole number knowledge is beneficial for math achievement, we found that whole number comparison performance was positively related to shared component fraction comparison performance (Level 1). This effect remained significant even after controlling for working memory and inhibitory control, indicating that whole number knowledge contributed over and above domain-general capacities. Interestingly, the effect of whole number knowledge increased with grade level, which may indicate a shift in strategy use. As students gain proficiency with shared component fractions, they may focus more on comparing the non-shared numbers, such that differences in whole number knowledge become more relevant.

This shift in strategy may help explain the three-way interaction between grade, congruency type, and whole number knowledge that we found for performance on shared component problems (Level 1). Specifically, for $3^{\text {rd }}$ grade, whole number comparison performance predicted fraction comparison on the same numerator problems but not the same denominator problems. There was also a trend in this direction for $5^{\text {th }}$ graders, but by $7^{\text {th }}$ grade, whole number knowledge predicted 
both congruency types comparably. This result is somewhat unexpected because whole number comparison is most similar to congruent, same denominator comparison, as only numerators must be compared. In contrast, for the incongruent, same numerator problems, the denominators must be compared, and the fraction with the smaller denominator is the larger fraction. Studies that include eye tracking could help elucidate this result by examining whether children with varying whole number knowledge focus differentially on the denominators versus numerators on these types of problems (Miller Singley et al., 2020).

For the more challenging distinct component fraction comparisons (Level 2), we found that whole number knowledge had no significant impact on performance. This lack of relation might reflect the fact that only higher performing students made it to Level 2, and thus there is less variance in the whole number comparison performance in these students (as well as a smaller sample size in which to detect an effect). However, we were still able to detect effects for inhibitory control at this level, suggesting that sampling bias does not preclude finding effects of these factors. Additional work is needed to assess how whole number knowledge relates to various types of fraction knowledge across the ability spectrum. Nevertheless, the lack of a positive contribution of whole number knowledge for fraction comparisons with distinct components suggests that whole number knowledge, as indexed by comparison tasks, may not be equally beneficial for all domains of mathematics.

Most prior work that has demonstrated the utility of whole number knowledge for fractions has employed number line estimation tasks rather than whole number comparison tasks. Indeed, several longitudinal studies employing number line estimation have shown that whole number knowledge is positively related to future performance on a variety of broad measures of rational number understanding, such as conceptual and procedural knowledge of fraction arithmetic 
(Bailey et al., 2014; Jordan et al., 2013; Van Hoof, Verschaffel, \& Van Dooren, 2017). The only study to our knowledge to collect both number line and whole number comparison found that number line estimation was a stronger predictor of rational number understanding than whole number comparison (Van Hoof et al., 2017). Number line tasks may better capture learners' understanding of numbers as quantities that represent magnitudes, whereas comparison tasks may index automatic access to numbers' ordinal information (Lyons \& Beilock, 2013). This view of number comparison may explain its contribution to same numerator problems, where instead of interfering with these supposedly contradictory problems, it indexes automatic access to ordinal information that can be used to quickly identify the smaller quantity. Although performance on whole number comparison and number line estimation tasks tend to be positively correlated (Laski \& Siegler, 2007), further research should include both measures in order to disentangle which aspects of whole number knowledge support rational number outcomes.

\section{The role of inhibitory control beyond whole number interference}

In the current study, we sought to test to predictions of the whole number interference hypothesis, namely that one source of difficulty with rational numbers is interference caused by whole number magnitude knowledge. One prediction from this hypothesis is that whole number knowledge should hinder fraction comparison performance because many properties of whole numbers do not apply to rational numbers. However, we did not observe a negative relation between whole number knowledge and fraction comparison performance, and furthermore, we did not find evidence for an interaction between whole number knowledge and inhibitory control. In other words, the participants with better whole number knowledge did not need more inhibitory control to overcome that knowledge. This pattern of results suggests that although inhibitory control 
contributes to fraction comparison performance, it is serving a function other than inhibiting whole number knowledge.

The performance patterns for the congruent versus incongruent problems with distinct components (Level 2) provides some insight into alternative contributions of inhibitory control. We hypothesized that congruency with whole number knowledge would be a salient factor that influences fraction comparison performance for problems with distinct components. By this logic, incongruent comparisons should be more difficult than congruent ones because they involve two sub-comparisons that contradict whole number knowledge (e.g., in the comparison of 2/3 vs. 5/9, the larger magnitude fraction contains both the smaller numerator and denominator components). Contrary to our expectations, participants were more accurate on the incongruent distinct component problems than on the congruent problems. However, a number of previous studies have documented stronger performance for incongruent relative to congruent problems with distinct components (Gómez \& Dartnell, 2018; González-Forte, Fernández, \& Van Dooren, 2018; González-Forte, Fernández, Van Hoof, \& Van Dooren, 2020; Obersteiner et al., 2013; Rinne et al., 2017; Toledo, Abreu-Mendoza, \& Rosenberg-Lee, 2022, August 10), which may reflect the strategy that participants engaged in when solving these problems. In these cases, participants (or at least a subset of participants), may have used a "select the smaller number strategy," which would lead to the correct answer for incongruent but not congruent problems. In fact, recent studies have identified this exact strategy among a subset of children (Gómez \& Dartnell, 2018; Miller Singley et al., 2020; Rinne et al., 2017). This strategy may reflect a transition from a more naïve "select the larger strategy" to a more sophisticated understanding of fractions, whereby students note that something is "different" about fractions and acknowledge that the presence of larger numbers is not necessarily an indicator of a larger magnitude (Rinne et al., 2017). In turn, students 
who have this understanding may be monitoring their responses more carefully, thus invoking inhibitory control for both congruency problem types. An important direction for future studies will be to move beyond simple accuracy measures and instead use participants' individual patterns of success and failure across problem types to identify different underlying strategies or learning profiles (Braithwaite, Leib, Siegler, \& McMullen, 2019).

Another possible source of performance differences between congruent and incongruent comparisons is in the properties of the fraction pairs. Constructing well-matched sets of congruent and incongruent fraction comparison pairs is difficult because controlling for one feature sometimes makes it mathematically difficulty to control for another feature (Rosenberg-Lee, 2021). In the present study, we chose to control for magnitude difference between the fraction pairs and partial distance. Although, we were not able to exactly match between the conditions on factors like gap distance, half benchmarking, simplified forms, and familiarity, which have been shown to impact performance (Clarke \& Roche, 2009; González-Forte et al., 2018; González-Forte et al., 2020), there does not seem to be a definitive impact of these factors on students' performance in the current sample (Table S3). For example, applying the gap strategy (selecting the fraction with the smallest distance between numerator and denominator) would only lead to an error on one congruent pair (2/7 vs 3/9), yet performance was no worse on this pair (see Table S3). Future studies should systematically manipulate these factors to explicitly test how inhibitory control relates to these stimulus features. It is also important to note that the fraction task used in this study was timed, whereas children and adults do not often have stringent time constraints in most real-world situations with fractions. Even though participants were not using the full RT window available, students may use different strategies when under time pressure compared to when they 
can pace themselves. Thus, another future direction is to compare the role of inhibitory control in timed and untimed fraction tasks.

Beyond the specifics of the fraction stimuli, the pattern of inhibitory control engagement found here is in line with prior work that suggests that learning about rational numbers requires conceptual change (McMullen, Laakkonen, Hannula-Sormunen, \& Lehtinen, 2015; McNeil \& Alibali, 2005; Vamvakoussi \& Vosniadou, 2004), and that conceptual change invokes inhibitory control (Bascandziev et al., 2018; Brookman-Byrne, Mareschal, Tolmie, \& Dumontheil, 2018). Specifically, in cases in which learners acquire new counter-intuitive knowledge that contradicts previous knowledge, learners maintain both conceptual frameworks and inhibitory control is required to activate the correct knowledge and inhibit the initial, inappropriate knowledge (Shtulman \& Valcarcel, 2012; Vosniadou, 2014). In the case of fractions, what therefore needs to be inhibited may not be specifically knowledge of whole number magnitudes, but rather the entire conceptual framework of number properties that apply to whole numbers but not to rational numbers.

\section{Conclusions}

Consistent with a more general proposal that overcoming interference is a key building block for mastering rational numbers (Rosenberg-Lee, 2021), we found that individual differences in inhibitory control predicted children's fraction comparison performance. Further, this effect was independent of the contribution of working memory. However, contrary to the counterintuitive prediction about the role of whole number knowledge, we did not find that superior whole number knowledge hindered fraction understanding. Instead, whole number knowledge positively predicted performance for the easier, shared component problems and was not related to performance on distinct component problems. Further, we found no differences in these patterns 
between congruent and incongruent problems, and developmentally, the contributions of these factors were generally stable from $3^{\text {rd }}$ to $7^{\text {th }}$ grade. Together, these results converge with the growing body of evidence pointing to a role for inhibitory control in rational number understanding (Abreu-Mendoza et al., 2020; Avgerinou \& Tolmie, 2019; Coulanges et al., 2021; Gómez et al., 2015; Ren \& Gunderson, 2021), and further suggest that its contribution may be more general than overcoming whole number magnitude knowledge. Given that individual differences in inhibitory control are evident far before children are exposed to formal fraction instruction, assessing this capacity early on could be useful to identify students who are likely to benefit from additional support while learning fractions. 


\section{References}

Abreu-Mendoza, R. A., Chamorro, Y., Garcia-Barrera, M. A., \& Matute, E. (2018). The contributions of executive functions to mathematical learning difficulties and mathematical talent during adolescence. PLoS ONE, 13(12), e0209267. doi:10.1371/journal.pone.0209267

Abreu-Mendoza, R. A., Coulanges, L., Ali, K., Powell, A. B., \& Rosenberg-Lee, M. (2020). Children's discrete proportional reasoning is related to inhibitory control and enhanced by priming continuous representations. J Exp Child Psychol, 199, 104931. doi:10.1016/j.jecp.2020.104931

Allen, M., Poggiali, D., Whitaker, K., Marshall, T. R., Van Langen, J., \& Kievit, R. A. (2021). Raincloud plots: a multi-platform tool for robust data visualization. Wellcome Open Research, 4, 63. doi:10.12688/wellcomeopenres.15191.2

Avgerinou, V. A., \& Tolmie, A. (2019). Inhibition and cognitive load in fractions and decimals. The British journal of educational psychology. doi:10.1111/bjep.12321

Bailey, D. H., Siegler, R. S., \& Geary, D. C. (2014). Early predictors of middle school fraction knowledge. Developmental Science, 17(5), 775-785. doi:10.1111/desc.12155

Bascandziev, I., Tardiff, N., Zaitchik, D., \& Carey, S. (2018). The role of domain-general cognitive resources in children's construction of a vitalist theory of biology. Cogn Psychol, 104, 1-28. doi:10.1016/j.cogpsych.2018.03.002

Best, J. R., Miller, P. H., \& Naglieri, J. A. (2011). Relations between Executive Function and Academic Achievement from Ages 5 to 17 in a Large, Representative National Sample. Learn Individ Differ, 21(4), 327-336. doi:10.1016/j.lindif.2011.01.007

Bonato, M., Fabbri, S., Umiltà, C., \& Zorzi, M. (2007). The mental representation of numerical fractions: Real or integer? Journal of Experimental Psychology: Human Perception and Performance, 33(6), 1410-1419. doi:10.1037/0096-1523.33.6.1410

Braithwaite, D. W., Leib, E. R., Siegler, R. S., \& McMullen, J. (2019). Individual differences in fraction arithmetic learning. Cogn Psychol, 112, 81-98. doi:10.1016/j.cogpsych.2019.04.002

Braithwaite, D. W., \& Siegler, R. S. (2018). Developmental changes in the whole number bias. Developmental Science, 21(2), 13. doi:10.1111/desc.12541

Brookman-Byrne, A., Mareschal, D., Tolmie, A. K., \& Dumontheil, I. (2018). Inhibitory control and counterintuitive science and maths reasoning in adolescence. PLOS ONE, 13(6). doi:10.1371/journal.pone.0198973

Bugden, S., \& Ansari, D. (2011). Individual differences in children's mathematical competence are related to the intentional but not automatic processing of Arabic numerals. Cognition, 118(1), 32-44. doi:10.1016/j.cognition.2010.09.005

Bull, R., \& Lee, K. (2014). Executive Functioning and Mathematics Achievement. Child Development Perspectives, 8(1), 36-41. doi:10.1111/cdep.12059

Carey, S. (2011). Precis of 'The Origin of Concepts'. Behav Brain Sci, 34(3), 113-124; discussion 124-162. doi:10.1017/S0140525X10000919

Clarke, D. M., \& Roche, A. (2009). Students' fraction comparison strategies as a window into robust understanding and possible pointers for instruction. Educational Studies in Mathematics, 72(1), 127-138. doi:10.1007/s10649-009-9198-9

Constantinidis, C., \& Luna, B. (2019). Neural Substrates of Inhibitory Control Maturation in Adolescence. Trends in Neurosciences, 42(9), 604-616. doi:10.1016/j.tins.2019.07.004 
Corsi, P. (1972). HUMAN MEMORY AND THE MEDIAL TEMPORAL REGION OF THE BRAIN. In: ProQuest Dissertations Publishing.

Coulanges, L., Abreu-Mendoza, R. A., Varma, S., Uncapher, M. R., Gazzaley, A., Anguera, J., \& Rosenberg-Lee, M. (2021). Linking inhibitory control to math achievement via comparison of conflicting decimal numbers. Cognition, 214, 104767. doi:10.1016/j.cognition.2021.104767

De Smedt, B., Noël, M.-P., Gilmore, C., \& Ansari, D. (2013). How do symbolic and nonsymbolic numerical magnitude processing skills relate to individual differences in children's mathematical skills? A review of evidence from brain and behavior. Trends in Neuroscience and Education, 2(2), 48-55. doi:10.1016/j.tine.2013.06.001

DeWolf, M., \& Vosniadou, S. (2015). The representation of fraction magnitudes and the whole number bias reconsidered. Learning and Instruction, 37, 39-49. doi:10.1016/j.learninstruc.2014.07.002

Diamond, A. (2013). Executive functions. Annu Rev Psychol, 64, 135-168. doi:10.1146/annurevpsych-113011-143750

Draheim, C., Mashburn, C. A., Martin, J. D., \& Engle, R. W. (2019). Reaction time in differential and developmental research: A review and commentary on the problems and alternatives. Psychol Bull, 145(5), 508-535. doi:10.1037/bul0000192

Eriksen, B. A., \& Eriksen, C. W. (1974). Effects of noise letters upon the identification of a target letter in a nonsearch task. Perception \& Psychophysics, 16(1), 143-149. doi:10.3758/BF03203267

Farrell Pagulayan, K., Busch, R. M., Medina, K. L., Bartok, J. A., \& Krikorian, R. (2006). Developmental Normative Data for the Corsi Block-Tapping Task. Journal of Clinical and Experimental Neuropsychology, 28(6), 1043-1052. doi:10.1080/13803390500350977

Fazio, L. K., DeWolf, M., \& Siegler, R. S. (2016). Strategy Use and Strategy Choice in Fraction Magnitude Comparison. Journal of Experimental Psychology-Learning Memory and Cognition, 42(1), 1-16. doi:10.1037/xlm0000153

Friedman, N. P., \& Miyake, A. (2004). The Relations Among Inhibition and Interference Control Functions: A Latent-Variable Analysis. Journal of Experimental Psychology: General, 133(1), 101-135. doi:10.1037/0096-3445.133.1.101

Friso-van den Bos, I., van der Ven, S. H. G., Kroesbergen, E. H., \& van Luit, J. E. H. (2013). Working memory and mathematics in primary school children: A meta-analysis. Educational Research Review, 10, 29-44. doi:10.1016/j.edurev.2013.05.003

Fu, X., Li, X., Xu, P., \& Zeng, J. (2020). Inhibiting the Whole Number Bias in a Fraction Comparison Task: An Event-Related Potential Study. Psychology Research and Behavior Management, 13, 245-255. doi:10.2147/PRBM.S240263

Gómez, D. M., \& Dartnell, P. (2018). Middle Schoolers' Biases and Strategies in a Fraction Comparison Task. International Journal of Science and Mathematics Education, 17(6), 1233-1250. doi:10.1007/s10763-018-9913-z

Gómez, D. M., Jiménez, A., Bobadilla, R., Reyes, C., \& Dartnell, P. (2015). The effect of inhibitory control on general mathematics achievement and fraction comparison in middle school children. $Z d m$, 47(5), 801-811. doi:10.1007/s11858-015-0685-4

González-Forte, J. M., Fernández, C., \& Van Dooren, W. (2018, 2018). Gap and congruency effect in fraction comparison. Paper presented at the Proceedings of the 42nd Conference of the International Group for the Psychology of Mathematics Education, Umeå, Sweden. 
González-Forte, J. M., Fernández, C., Van Hoof, J., \& Van Dooren, W. (2020). Various ways to determine rational number size: an exploration across primary and secondary education. European Journal of Psychology of Education, 35(3), 549-565. doi:10.1007/s10212-01900440-w

Hedge, C., Powell, G., \& Sumner, P. (2018). The reliability paradox: Why robust cognitive tasks do not produce reliable individual differences. Behav Res Methods, 50(3), 1166-1186. doi:10.3758/s 13428-017-0935-1

Ischebeck, A., Schocke, M., \& Delazer, M. (2009). The processing and representation of fractions within the brain: an fMRI investigation. Neuroimage, 47(1), 403-413. doi:10.1016/j.neuroimage.2009.03.041

Jordan, N. C., Hansen, N., Fuchs, L. S., Siegler, R. S., Gersten, R., \& Micklos, D. (2013). Developmental predictors of fraction concepts and procedures. Journal of Experimental Child Psychology, 116(1), 45-58. doi:10.1016/j.jecp.2013.02.001

Kainulainen, M., McMullen, J., \& Lehtinen, E. (2017). Early Developmental Trajectories Toward Concepts of Rational Numbers. Cognition and Instruction, 35(1), 4-19. doi:10.1080/07370008.2016.1251287

Kuznetsova, A., Brockhoff, P. B., \& Christensen, R. H. B. (2017). lmerTest Package: Tests in Linear Mixed Effects Models. Journal of Statistical Software, 82(13), 1-26. doi:DOI 10.18637/jss.v082.i13

Laski, E. V., \& Siegler, R. S. (2007). Is 27 a big number? Correlational and causal connections among numerical categorization, number line estimation, and numerical magnitude comparison Child Development, 78(6), 1723-1743. Retrieved from https://onlinelibrary.wiley.com/doi/pdf/10.1111/j.1467-8624.2007.01087.x

Lawson, G. M., \& Farah, M. J. (2017). Executive function as a mediator between SES and academic achievement throughout childhood. International Journal of Behavioral Development, 41(1), 94-104. doi:10.1177/0165025415603489

Lee, K., \& Bull, R. (2016). Developmental Changes in Working Memory, Updating, and Math Achievement. Journal of Educational Psychology, 108(6), 869-882. doi:10.1037/edu0000090

Lee, K., \& Lee, H. W. (2019). Inhibition and Mathematical Performance: Poorly Correlated, Poorly Measured, or Poorly Matched? Child Development Perspectives, 13(1), 28-33. doi:10.1111/cdep.12304

Lyons, I. M., \& Beilock, S. L. (2013). Ordinality and the nature of symbolic numbers. The Journal of neuroscience : the official journal of the Society for Neuroscience, 33(43), 17052-17061. doi:10.1523/JNEUROSCI.1775-13.2013

Matthews, P. G., Lewis, M. R., \& Hubbard, E. M. (2016). Individual Differences in Nonsymbolic Ratio Processing Predict Symbolic Math Performance. Psychological Science, 27(2), 191-202. doi:10.1177/0956797615617799

McMullen, J., Laakkonen, E., Hannula-Sormunen, M., \& Lehtinen, E. (2015). Modeling the developmental trajectories of rational number concept(s). Learning and Instruction, 37, 14-20. doi:10.1016/j.learninstruc.2013.12.004

McNeil, N. M., \& Alibali, M. W. (2005). Why won't you change your mind? Knowledge of operational patterns hinders learning and performance on equations. Child Dev, 76(4), 883-899. doi:10.1111/j.1467-8624.2005.00884.x

Mead, L. A., Mayer, A. R., Bobholz, J. A., Woodley, S. J., Cunningham, J. M., Hammeke, T. A., $\&$ Rao, S. M. (2002). Neural basis of the Stroop interference task: Response competition 
or selective attention? Journal of the International Neuropsychological Society, 8(6), 735-742. doi:10.1017/S1355617702860015

Meert, G., Gregoire, J., \& Noel, M. P. (2009). Rational numbers: componential versus holistic representation of fractions in a magnitude comparison task. Q J Exp Psychol (Colchester), 62(8), 1598-1616. doi:10.1080/17470210802511162

Meert, G., Gregoire, J., \& Noel, M. P. (2010). Comparing the magnitude of two fractions with common components: which representations are used by 10 - and 12-year-olds? J Exp Child Psychol, 107(3), 244-259. doi:10.1016/j.jecp.2010.04.008

Miller Singley, A. T., \& Bunge, S. A. (2018). Eye gaze patterns reveal how we reason about fractions. Thinking \& Reasoning, 24(4), 445-468. doi:10.1080/13546783.2017.1417909

Miller Singley, A. T., Crawford, J. L., \& Bunge, S. A. (2020). Eye gaze patterns reflect how young fraction learners approach numerical comparisons. Journal of Numerical Cognition, 6(1), 83-107. doi:10.5964/jnc.v6i1.119

National Governors Association Center for Best Practices, \& Council of Chief State School Officers. (2010). Common Core State Standards for Mathematics. In. Washington, DC: Authors.

Ni, Y., \& Zhou, Y.-D. (2005). Teaching and Learning Fraction and Rational Numbers: The Origins and Implications of Whole Number Bias. Educational Psychologist, 40(1), 27-52. doi:10.1207/s15326985ep4001_3

Obersteiner, A., Van Dooren, W., Van Hoof, J., \& Verschaffel, L. (2013). The natural number bias and magnitude representation in fraction comparison by expert mathematicians. Learning and Instruction, 28, 64-72. doi:10.1016/j.learninstruc.2013.05.003

Opfer, J. E., \& Siegler, R. S. (2007). Representational change and children's numerical estimation Cognitive Psychology, 55(3), 169-195. Retrieved from https://ac.elscdn.com/S0010028506000685/1-s2.0-S0010028506000685-main.pdf?_tid=ea0b1514$5 \mathrm{~b} 6 \mathrm{c}-4234-\mathrm{b} 048-$ e1e6749e1781\&acdnat $=1545338239$ c6907cb3ece5bd58e3143bdb69c5e7b6

Park, Y., \& Matthews, P. G. (2021). Revisiting and refining relations between nonsymbolic ratio processing and symbolic math achievement. Journal of Numerical Cognition, 7(3), 328350. doi:10.5964/jnc.6927

Peng, P., Namkung, J., Barnes, M., \& Sun, C. (2016). A Meta-Analysis of Mathematics and Working Memory: Moderating Effects of Working Memory Domain, Type of Mathematics Skill, and Sample Characteristics. Journal of Educational Psychology, 108(4), 455-473. doi:10.1037/edu0000079

R Core Team. (2019). R: A language and environment for statistical computing. Retrieved from https://www.R-project.org

Ramani, G. B., \& Siegler, R. S. (2008). Promoting broad and stable improvements in lowincome children's numerical knowledge through playing number board games. Child Development, 79(2), 375-394. doi:DOI 10.1111/j.1467-8624.2007.01131.x

Ren, K. X., \& Gunderson, E. A. (2021). The dynamic nature of children's strategy use after receiving accuracy feedback in decimal comparisons. Journal of Experimental Child Psychology, 202. doi:10.1016/j.jecp.2020.105015

Rinne, L. F., Ye, A., \& Jordan, N. C. (2017). Development of Fraction Comparison Strategies: A Latent Transition Analysis. Developmental Psychology, 53(4), 713-730. doi:10.1037/dev0000275 
Rose, S. A., Feldman, J. F., \& Jankowski, J. J. (2011). Modeling a cascade of effects: the role of speed and executive functioning in preterm/full-term differences in academic achievement. Developmental Science, 14(5), 1161-1175. doi:10.1111/j.14677687.2011.01068.x

Rosenberg-Lee, M. (2021). Probing the neural basis rational number difficulties: the role of inhibitory control and magnitude processing. In A. Henik \& W. Fias (Eds.), Learning and Education in Numerical Cognition: Elsevier.

Rossi, S., Vidal, J., Letang, M., Houdé, O., \& Borst, G. (2019). Adolescents and adults need inhibitory control to compare fractions. Journal of Numerical Cognition, 5(3), 314-336. doi:10.5964/jnc.v5i3.197

Schneider, M., Beeres, K., Coban, L., Merz, S., Susan Schmidt, S., Stricker, J., \& De Smedt, B. (2017). Associations of non-symbolic and symbolic numerical magnitude processing with mathematical competence: a meta-analysis. Developmental Science, 20(3), 1-16. doi:10.1111/desc. 12372

Shtulman, A., \& Valcarcel, J. (2012). Scientific knowledge suppresses but does not supplant earlier intuitions. Cognition, 124(2), 209-215. doi:10.1016/j.cognition.2012.04.005

Siegler, R. S., Duncan, G. J., Davis-Kean, P. E., Duckworth, K., Claessens, A., Engel, M., Susperreguy, M. I., \& Chen, M. C. (2012). Early Predictors of High School Mathematics Achievement. Psychological Science, 23(7), 691-697. doi:10.1177/0956797612440101

Siegler, R. S., Thompson, C. A., \& Schneider, M. (2011). An integrated theory of whole number and fractions development. Cognitive Psychology, 62(4), 273-296. doi:10.1016/j.cogpsych.2011.03.001

Singley, A. T. M., \& Bunge, S. A. (2018). Eye gaze patterns reveal how we reason about fractions. Thinking \& Reasoning, 24(4), 445-468. doi:10.1080/13546783.2017.1417909

Starr, A., Leib, E. R., Younger, J. W., Uncapher, M. R., \& Bunge, S. A. (2022). Relational thinking: An overlooked component of executive functioning. Developmental Science. doi:10.1111/desc. 13320

Stricker, J., Vogel, S. E., Schoneburg-Lehnert, S., Krohn, T., Dognitz, S., Jud, N., Spirk, M., Windhaber, M. C., Schneider, M., \& Grabner, R. H. (2021). Interference between naive and scientific theories occurs in mathematics and is related to mathematical achievement. Cognition, 214, 104789. doi:10.1016/j.cognition.2021.104789

Stroop, J. R. (1935). Studies of interference in serial verbal reactions. Journal of Experimental Psychology, 18, 643-662. doi:DOI 10.1037/h0054651

Toledo, R. V. F., Abreu-Mendoza, R. A., \& Rosenberg-Lee, M. (2022, August 10). Brazilian math teacher's magnitude representation and strategy use in fraction comparison: a mixed methods study. PsychArXiv Preprints. doi:10.31234/osf.io/yrngz

Vamvakoussi, X., \& Vosniadou, S. (2004). Understanding the structure of the set of rational numbers: a conceptual change approach. Learning and Instruction, 14(5), 453-467. doi:10.1016/j.learninstruc.2004.06.013

Van Dooren, W., \& Inglis, M. (2015). Inhibitory control in mathematical thinking, learning and problem solving: a survey. Zdm-Mathematics Education, 47(5), 713-721. doi:10.1007/s11858-015-0715-2

Van Hoof, J., Ceulemans, E., \& Van Dooren, W. (2021). The Role of the Inhibition of Natural Number Based Reasoning and Strategy Switch Cost in a Fraction Comparison Task. Studia Psychologica, 63(1), 64-76. doi:10.31577/sp.2021.01.814 
Van Hoof, J., Degrande, T., Ceulemans, E., Verschaffel, L., \& Van Dooren, W. (2018). Towards a mathematically more correct understanding of rational numbers: A longitudinal study with upper elementary school learners. Learning and Individual Differences, 61, 99-108. doi:10.1016/j.lindif.2017.11.010

Van Hoof, J., Verschaffel, L., \& Van Dooren, W. (2017). Number sense in the transition from natural to rational numbers. The British journal of educational psychology, 87(1), 43-56. doi:10.1111/bjep.12134

Vandierendonck, A. (2017). A comparison of methods to combine speed and accuracy measures of performance: A rejoinder on the binning procedure. Behav Res Methods, 49(2), 653673. doi:10.3758/s13428-016-0721-5

Vosniadou, S. (2014). Examining cognitive development from a conceptual change point of view: The framework theory approach. European Journal of Developmental Psychology, 11(6), 645-661. doi:10.1080/17405629.2014.921153

Wickham, H. (2016). ggplot2: Elegant Graphics for Data Analysis: Springer-Verlag New York. Retrieved from https://ggplot2.tidyverse.org

Woltz, D. J., \& Was, C. A. (2006). Availability of related long-term memory during and after attention focus in working memory. Memory \& Cognition, 34(3), 668-684. doi:10.3758/BF03193587

Younger, J., O'Laughlin, K. D., Anguera, J., Bunge, S., Ferrer, E., Hoeft, F., McCandliss, B. D., Mishra, J., Rosenberg-Lee, M., Gazzaley, A., \& Uncapher, M. R. (2021). Development of Executive Function in Middle Childhood: a Large-Scale, In-School, Longitudinal Investigation [Preprint]. PsyArXiv. doi:https://doi.org/10.31234/osf.io/xf489

Younger, J. W., O'Laughlin, K. D., Anguera, J. A., Bunge, S. A., Ferrer, E., Hoeft, F., McCandliss, B. D., Mishra, J., Rosenberg-Lee, M., Gazzaley, A., \& Uncapher, M. R. (2022). Better together: Novel methods for measuring and modeling development of executive function diversity while accounting for unity doi:10.31234/osf.io/ctzwx

Zhang, L., Fang, Q., Gabriel, F. C., \& Szucs, D. (2014). The componential processing of fractions in adults and children: effects of stimuli variability and contextual interference. Frontiers in psychology, 5, 981. doi:10.3389/fpsyg.2014.00981 